STUPP-12-209

June, 2012

\title{
Coupling of the BLG theory to a conformal supergravity background
}

\author{
Madoka Nishimura $^{\mathrm{a}}$ and Yoshiaki Tanit ${ }^{\mathrm{t}}{ }^{*}$ \\ ${ }^{a}$ Department of Community Service and Science \\ Tohoku University of Community Service and Science \\ Iimoriyama 3-5-1, Sakata 998-8580, Japan \\ ${ }^{\mathrm{b}}$ Division of Material Science \\ Graduate School of Science and Engineering \\ Saitama University, Saitama 338-8570, Japan
}

\begin{abstract}
The Bagger-Lambert-Gustavsson theory is coupled to an off-shell $D=$ $3, \mathcal{N}=8$ conformal supergravity background. Local transformation laws of the conformal supergravity multiplet are obtained from those of the $D=4, \mathcal{N}=8 \mathrm{SO}(8)$ gauged supergravity. The complete Lagrangian and local transformation laws of the coupled theory are obtained. As an application the supercurrent multiplet in a flat background is obtained.
\end{abstract}

\footnotetext{
*e-mail: tanii@phy.saitama-u.ac.jp
} 


\section{Introduction}

The purpose of this paper is to study a coupling of the Bagger-Lambert-Gustavsson (BLG) theory [1-3] to a conformal supergravity background. The BLG theory is a three-dimensional field theory which was proposed to be related to a world-volume theory of M2-branes. (For a review, see [4].) It is invariant under $\mathcal{N}=8$ superconformal transformations and is naturally coupled to $D=3, \mathcal{N}=8$ conformal supergravity.

Our main motivation for studying such a coupling comes from the AdS/CFT correspondence [5]. In the AdS/CFT correspondence superstring/M-theory or its low energy effective supergravity theory in $(d+1)$-dimensional anti de Sitter (AdS) spacetime times a compact manifold is dual to a conformal field theory on $d$-dimensional boundary at infinity. Boundary values of the supergravity fields play a role of sources for operators of the conformal field theory [6,7]. These boundary supergravity fields at infinity form a $d$-dimensional conformal supergravity multiplet [8,9]. Local transformations of the $(d+1)$-dimensional supergravity induce local transformations of the $d$-dimensional conformal supergravity on these fields [10 13]. A typical example of the AdS/CFT correspondence is the correspondence between type IIB superstring or supergravity on $\mathrm{AdS}_{5} \times \mathrm{S}^{5}$ and $D=4, \mathcal{N}=4$ super Yang-Mills theory. The latter theory is invariant under $\mathcal{N}=4$ superconformal transformations and can be coupled to $D=4, \mathcal{N}=4$ conformal supergravity [14. Similarly, the BLG theory, which is supposed to be dual to M-theory on $\mathrm{AdS}_{4} \times S^{7}$, can be coupled to $D=3, \mathcal{N}=8$ conformal supergravity.

Apart from a relation to the AdS/CFT correspondence the coupling of the BLG theory to a background conformal supergravity is useful when studying its field theoretical properties. Conformal supergravity fields couple to operators in the supercurrent multiplet of the BLG theory such as the energy-momentum tensor and the supercurrents. One can use the conformal supergravity fields as sources to treat these operators systematically. The coupled theory can be used also to obtain a field theory with a rigid supersymmetry in a curved spacetime [15] by choosing a background invariant under supertransformations.

A coupling of the BLG theory to $D=3, \mathcal{N}=8$ conformal supergravity was previously studied in [16,17]. The conformal supergravity multiplet considered there in the component field formulation contains a dreibein, eight Rarita-Schwinger fields and $\mathrm{SO}(8)$ gauge fields. Local symmetry transformation laws of these fields were 
obtained, and an invariant Lagrangian of the coupled theory was constructed. The Lagrangian contains the kinetic terms of the conformal supergravity multiplet, and commutators of local supertransformations close only when their field equations are used. In this sense the conformal supergravity multiplet considered in [16, 17] is an on-shell multiplet.

Since conformal supergravity we consider in this paper is a non-dynamical background, the commutator algebra of local transformations should close off-shell. Such an off-shell $D=3, \mathcal{N}=8$ conformal supergravity multiplet was discussed in the superspace formulation in [18, 19, 17]. The off-shell multiplet contains scalar and spinor fields in addition to the fields of the on-shell multiplet. Local supertransformation laws of the component fields were explicitly given at the linearized level in [20]. In this paper we will obtain a nonlinear form of local transformation laws of the off-shell multiplet in the component field formulation. There are two kinds of conformal supergravity multiplets depending on a sign parameter $\eta= \pm 1$ appearing in self-duality conditions on the scalar fields. Then, we will construct a complete Lagrangian of the BLG theory coupled to the off-shell conformal supergravity background in the $\eta=+1$ case. In the $\eta=-1$ case we did not succeed in constructing a coupled theory. As we will discuss in section 6, the coupled theory studied in the previous works [16, 17] corresponds to the $\eta=-1$ case. Therefore, our coupled theory for $\eta=+1$ is the case not considered previously. As an application of the coupled theory we will discuss the supercurrent multiplet of the BLG theory in a flat background. Knowledge on off-shell conformal supergravities may be also useful to construct new supergravity theories [20].

The organization of this paper is as follows. In the next section we briefly explain how to find local transformations of the off-shell conformal supergravity multiplet starting from the $D=4, \mathcal{N}=8 \mathrm{SO}(8)$ gauged supergravity. Explicit forms of the local transformations are given in section 3. In section 4 we obtain local symmetry transformations and an invariant Lagrangian of the BLG theory coupled to the conformal supergravity background. In section 5 we study the supercurrent multiplet of the BLG theory in a flat background. In section 6 we discuss difficulties in the $\eta=-1$ case, comparisons with the previous works, and truncations of the $\mathcal{N}=8$ conformal supergravity multiplet to lower $\mathcal{N}$. In Appendix A we explain our notations and conventions. In Appendix B we collect identities of $\mathrm{SO}(8)$ matrices, which are useful for calculations in the text. 


\section{Conformal supergravity from gauged supergravity}

In this section we briefly sketch how to derive local transformation laws of the $D=3, \mathcal{N}=8$ conformal supergravity from those of the $D=4, \mathcal{N}=8 \mathrm{SO}(8)$ gauged supergravity, which has a four-dimensional AdS solution. Our approach is the same as those in [10 13] and is based on an idea in the AdS/CFT correspondence [5-7]. We study boundary behaviors of the $D=4$ gauged supergravity at infinity of fourdimensional spacetime. Boundary values of the fields form a $D=3$ conformal supergravity multiplet. We obtain local transformations on these three-dimensional fields induced from the four-dimensional local transformations. The resulting local transformation laws of the $D=3, \mathcal{N}=8$ conformal supergravity are given in the next section in a self-contained way.

The field content of the $D=4, \mathcal{N}=8 \mathrm{SO}(8)$ gauged supergravity [21] is a vierbein $e_{M}^{A}(x), 8$ Majorana Rarita-Schwinger fields $\psi_{M}^{\alpha}(x), 28 \mathrm{SO}(8)$ gauge fields $B_{M}^{\alpha \beta}(x)=-B_{M}^{\beta \alpha}(x), 56$ Majorana spinor fields $\lambda^{\alpha \beta \gamma}(x)=\lambda^{[\alpha \beta \gamma]}(x)$ and 70 real scalar fields. Here, $M, N, \cdots=0,1,2,3$ and $A, B, \cdots=0,1,2,3$ are four-dimensional world and local Lorentz indices respectively, and $\alpha, \beta, \gamma, \cdots=1,2, \cdots, 8$ are $\mathrm{SO}(8)$ spinor indices of negative chirality. (See Appendix A for our notations and conventions.) The scalar fields take values in the coset space $\mathrm{E}_{7(+7)} / \mathrm{SU}(8)[22]$ and can be represented by a $56 \times 56$ matrix

$$
V(x)=\exp \left(\begin{array}{cc}
0 & \frac{1}{\sqrt{2}} \phi(x) \\
\frac{1}{\sqrt{2}} \phi(x)^{*} & 0
\end{array}\right),
$$

where $\phi(x)$ is a complex $28 \times 28$ matrix. Components of $\phi(x)$ are denoted as $\phi^{\alpha \beta \gamma \delta}(x)$, where the antisymmetrized pairs of indices $[\alpha \beta]$ and $[\gamma \delta]$ label rows and columns, respectively. $\phi^{\alpha \beta \gamma \delta}(x)$ is totally antisymmetric in four indices and satisfies a selfduality condition

$$
\phi^{\alpha_{1} \cdots \alpha_{4}}=\frac{1}{4 !} \eta \epsilon^{\alpha_{1} \cdots \alpha_{8}}\left(\phi^{\alpha_{5} \cdots \alpha_{8}}\right)^{*},
$$

where $\epsilon^{\alpha_{1} \cdots \alpha_{8}}$ is the totally antisymmetric symbol with $\epsilon^{12345678}=+1$ and $\eta$ is a parameter taking a value +1 or -1 . Local symmetries of this theory are those under general coordinate transformations, local Lorentz transformations, $\mathrm{SO}(8)$ gauge transformations and local supertransformations.

Field equations of this theory have a solution for which the metric is given by AdS spacetime and other fields are zero. The four-dimensional AdS spacetime is locally 
represented as a region $r \equiv x^{3}>0$ in $\mathbb{R}^{4}$ with coordinates $x^{M}$. The boundary at infinity of the AdS spacetime corresponds to a hyperplane $r=0$. The AdS metric is given by

$$
d x^{M} d x^{N} \bar{g}_{M N}=\frac{1}{(2 g r)^{2}}\left(d r^{2}+d x^{\mu} d x^{\nu} \eta_{\mu \nu}\right)
$$

where $\mu, \nu=0,1,2$ are three-dimensional world indices and $g$ is the $\mathrm{SO}(8)$ gauge coupling constant. We will consider fluctuations of the fields around this solution.

To proceed we partially fix a gauge for the local symmetries. We choose gauge fixing conditions as

$$
e_{r}^{3}=\frac{1}{2 g r}, \quad e_{r}^{a}=0, \quad e_{\mu}^{3}=0, \quad \psi_{r}=0, \quad B_{r}^{\alpha \beta}=0,
$$

where $a, b, \cdots=0,1,2$ are three-dimensional local Lorentz indices. By solving linearized field equations we can find asymptotic behaviors of the fields near the boundary $r=0$. The boundary conditions will be chosen later such that they are consistent with these boundary behaviors. We assume that $e_{\mu}{ }^{a}$ behaves as $r^{-1}$ just as in the background (2.3). Boundary behaviors of other fields are determined by their linearized field equations around the background. For each field there are two independent solutions which have different boundary behaviors. For instance, the vector fields $B_{\mu}^{\alpha \beta}$ have two solutions which behave as $r^{0}$ and $r^{1}$ for $r \rightarrow 0$. The solution regular in the bulk is a linear combination of these two solutions and its boundary behavior is dominated by a solution which becomes larger near the boundary, i.e., $B_{\mu}^{\alpha \beta} \sim r^{0}$ in this case. To solve the fermionic field equations it is convenient to introduce projection operators $\frac{1}{2}\left(1 \pm \gamma^{3}\right)$, where $\gamma^{3}$ is a four-dimensional gamma matrix in the $A=3$ direction, and define $\psi_{\mu \pm}=\frac{1}{2}\left(1 \pm \gamma^{3}\right) \psi_{\mu}, \lambda_{ \pm}=\frac{1}{2}\left(1 \pm \gamma^{3}\right) \lambda$.

Knowing the boundary behaviors of the fields we impose Dirichlet type boundary conditions for $r \rightarrow 0$ as

$$
\begin{aligned}
e_{\mu}{ }^{a}(x, r) & \sim(2 g r)^{-1}{e_{0 \mu}}^{a}(x), \quad \psi_{0 \mu-}(x, r) \sim(2 g r)^{-\frac{1}{2}} \psi_{0 \mu-}(x), \\
B_{\mu}^{\alpha \beta}(x, r) & \sim g^{-1} B_{0 \mu}^{\alpha \beta}(x), \quad \lambda_{-}(x, r) \sim(2 r)^{\frac{3}{2}} g^{\frac{1}{2}} \lambda_{0-}(x), \\
\phi^{\alpha \beta \gamma \delta}(x, r) & \sim(2 r)^{2} D_{0}^{\alpha \beta \gamma \delta}(x)+2 i r E_{0}^{\alpha \beta \gamma \delta}(x),
\end{aligned}
$$

where $\Phi_{0}=\left(e_{0 \mu}{ }^{a}, \psi_{0 \mu-}, B_{0 \mu}, \lambda_{0-}, D_{0}, E_{0}\right)$ are arbitrary fixed functions on the boundary. The real scalar fields $D_{0}^{\alpha \beta \gamma \delta}, E_{0}^{\alpha \beta \gamma \delta}$ are totally antisymmetric in four indices and 
satisfy (anti) self-duality conditions

$$
\begin{aligned}
& D_{0}^{\alpha_{1} \cdots \alpha_{4}}=\frac{1}{4 !} \eta \epsilon^{\alpha_{1} \cdots \alpha_{8}} D_{0}^{\alpha_{5} \cdots \alpha_{8}}, \\
& E_{0}^{\alpha_{1} \cdots \alpha_{4}}=-\frac{1}{4 !} \eta \epsilon^{\alpha_{1} \cdots \alpha_{8}} E_{0}^{\alpha_{5} \cdots \alpha_{8}} .
\end{aligned}
$$

Note that we imposed the boundary conditions on only half of components of $\psi_{\mu}$ and $\lambda$ since their field equations are first order. Other components of these fields behave as $\psi_{\mu+}(x, r) \sim(2 r)^{\frac{1}{2}} g^{-\frac{1}{2}} \psi_{0 \mu+}(x), \lambda_{+}(x, r) \sim(2 r)^{\frac{5}{2}} g^{\frac{1}{2}} \lambda_{0+}(x)$. By the field equations $\psi_{0 \mu+}, \lambda_{0+}$ can be expressed in terms of $\Phi_{0}$ (see (3.5)) and therefore cannot be chosen arbitrarily. The fields $\Phi_{0}$ coincide with the field content of the off-shell $D=3, \mathcal{N}=8$ conformal supergravity [18,20].

We now study how the fields $\Phi_{0}$ transform under residual symmetry transformations after the gauge fixing. We first obtain parameters of residual symmetries for general coordinate, local Lorentz, $\mathrm{SO}(8)$ gauge and local super transformations, which preserve the gauge conditions (2.4). Parameters of these transformations are denoted as $\xi^{M}, \lambda^{A B}=-\lambda^{B A}, \zeta^{\alpha \beta}=-\zeta^{\beta \alpha}$ and $\epsilon$, respectively. By solving differential equations on these parameters obtained by taking variations of the gauge conditions (2.4) under the local transformations we find the parameters of the residual symmetry transformations near the boundary $r=0$ as

$$
\begin{aligned}
\xi^{\mu}(x, r) & =\xi_{0}^{\mu}(x)+\mathcal{O}\left(r^{2}\right), \\
\xi^{r}(x, r) & =-r \Lambda_{0}(x), \\
\lambda_{a b}(x, r) & =\lambda_{0 a b}(x)+\mathcal{O}\left(r^{2}\right), \\
\lambda_{a 3}(x, r) & =\mathcal{O}(r), \\
\zeta^{\alpha \beta}(x, r) & =\zeta_{0}^{\alpha \beta}(x)+\mathcal{O}\left(r^{2}\right), \\
\epsilon_{-}(x, r) & =(2 g r)^{-\frac{1}{2}}\left[\epsilon_{0-}(x)+\mathcal{O}\left(r^{2}\right)\right], \\
\epsilon_{+}(x, r) & =-(2 g)^{-\frac{1}{2}} r^{\frac{1}{2}}\left[\eta_{0+}(x)+\mathcal{O}\left(r^{2}\right)\right],
\end{aligned}
$$

where $\xi_{0}^{\mu}, \Lambda_{0}, \lambda_{0 a b}, \zeta_{0}^{\alpha \beta}, \epsilon_{0-}$ and $\eta_{0+}$ are arbitrary functions of $x^{\mu}(\mu=0,1,2)$. Order $\mathcal{O}(r)$ and $\mathcal{O}\left(r^{2}\right)$ terms are non-local functionals of these functions and the fields $\Phi_{0}$. The transformations with the parameters $\xi_{0}^{\mu}, \lambda_{0 a b}$ and $\epsilon_{0-}$ act on the boundary fields $\Phi_{0}$ as three-dimensional general coordinate, local Lorentz and local super transformations. The transformation with the parameter $\Lambda_{0}$ acts on the boundary fields as Weyl transformation. When a four-dimensional field behaves as $\varphi(x, r) \sim r^{-w} \varphi_{0}(x)$ for $r \rightarrow 0$ the transformation is $\delta \varphi_{0}(x)=w \Lambda_{0}(x) \varphi_{0}(x)$. The transformation with 
the parameter $\eta_{0}$ acts on the boundary fields as super Weyl transformation. All the transformations of $\Phi_{0}$ become independent of the $\mathrm{SO}(8)$ gauge coupling constant $g$ when we scale the fields and the transformation parameters by $g$ as in (2.5) and (2.7). Explicit forms of the transformations obtained in this way will be given in the next section.

The supertransformations of the $D=4$ gauged supergravity are non-polynomial in the scalar fields $\phi$ as can be seen in the expression (2.1). However, in the limit $r \rightarrow 0$ they become polynomials in $D_{0}$ and $E_{0}$. Similarly, although the supertransformations of the fermionic fields in the $D=4$ gauged supergravity contain terms quadratic in the fermionic fields, most of them go to zero in the limit $r \rightarrow 0$. Only remaining such higher Fermi terms are those appearing in supercovariant derivatives.

In the following we will suppress the subscript 0 for the boundary fields and the transformation parameters. Four-component spinors of $\mathrm{SO}(1,3)$ with a definite $\gamma^{3}$ eigenvalue will be expressed by two-component spinors of $\mathrm{SO}(1,2)$, and the subscripts \pm of $\psi_{0 \mu_{-}}, \lambda_{0-}, \epsilon_{0-}, \eta_{0+}$ will be suppressed.

\section{3. $D=3, \mathcal{N}=8$ conformal supergravity}

Let us summarize the results on the $D=3, \mathcal{N}=8$ conformal supergravity obtained in the last section. The field content is, as given in Table 1, a dreibein $e_{\mu}{ }^{a}(x), 8$ Majorana Rarita-Schwinger fields $\psi_{\mu}^{\alpha}(x), 28 \mathrm{SO}(8)$ gauge fields $B_{\mu}^{\alpha \beta}(x)$, 56 Majorana spinor fields $\lambda^{\alpha \beta \gamma}(x), 70$ real scalar fields $D^{\alpha \beta \gamma \delta}(x), E^{\alpha \beta \gamma \delta}(x)$, where $\alpha, \beta, \cdots=1,2, \cdots, 8$ are $\mathrm{SO}(8)$ negative chirality spinor indices. The fields $B_{\mu}^{\alpha \beta}(x)$, $\lambda^{\alpha \beta \gamma}(x), D^{\alpha \beta \gamma \delta}(x), E^{\alpha \beta \gamma \delta}(x)$ are totally antisymmetric in these indices. The scalar fields also satisfy (anti) self-duality conditions

$$
\begin{aligned}
D^{\alpha_{1} \cdots \alpha_{4}} & =\frac{1}{4 !} \eta \epsilon^{\alpha_{1} \cdots \alpha_{8}} D^{\alpha_{5} \cdots \alpha_{8}}, \\
E^{\alpha_{1} \cdots \alpha_{4}} & =-\frac{1}{4 !} \eta \epsilon^{\alpha_{1} \cdots \alpha_{8}} E^{\alpha_{5} \cdots \alpha_{8}},
\end{aligned}
$$

where $\eta$ is a parameter taking a value +1 or -1 .

Local symmetry transformations of these fields are general coordinate transformation $\delta_{G}$ with a parameter $\xi^{\mu}(x)$, local Lorentz transformation $\delta_{L}$ with a parameter $\lambda_{a b}(x)=-\lambda_{b a}(x)$, Weyl transformation $\delta_{W}$ with a parameter $\Lambda(x)$, local $\mathrm{SO}(8)$ 


\begin{tabular}{|c|c|c|c|c|c|c|}
\hline Field & $e_{\mu}{ }^{a}$ & $\psi_{\mu}^{\alpha}$ & $B_{\mu}^{\alpha \beta}$ & $\lambda^{\alpha \beta \gamma}$ & $D^{\alpha \beta \gamma \delta}$ & $E^{\alpha \beta \gamma \delta}$ \\
\hline Weyl weight & 1 & $\frac{1}{2}$ & 0 & $-\frac{3}{2}$ & -2 & -1 \\
\hline $\mathrm{SO}(8)$ representation & $\mathbf{1}$ & $\mathbf{8}_{s}$ & $\mathbf{2 8}$ & $\mathbf{5 6}_{s}$ & $\mathbf{3 5}_{v}$ or $\mathbf{3} \mathbf{5}_{c}$ & $\mathbf{3 5}_{c}$ or $\mathbf{3 5}_{v}$ \\
\hline
\end{tabular}

Table 1: The field content of the conformal supergravity multiplet. $\mathrm{SO}(8)$ representations of $D^{\alpha \beta \gamma \delta}$ and $E^{\alpha \beta \gamma \delta}$ are the first ones for $\eta=+1$ and the second ones for $\eta=-1$.

transformation $\delta_{g}$ with a parameter $\zeta^{\alpha \beta}(x)=-\zeta^{\beta \alpha}(x)$, local supertransformation $\delta_{Q}$ with a Majorana spinor parameter $\epsilon^{\alpha}(x)$ and super Weyl transformation $\delta_{S}$ with a Majorana spinor parameter $\eta^{\alpha}(x)$. Weyl weights and $\mathrm{SO}(8)$ representations of the fields are given in Table 1. (See Tables 36, 37 of [23] for naming of $\mathrm{SO}$ (8) representations.) Bosonic transformation laws other than the Weyl transformation are obvious from the index structure of the fields. For instance, the bosonic transformations of the Rarita-Schwinger fields with Weyl weight $\frac{1}{2}$ are

$$
\left(\delta_{G}+\delta_{L}+\delta_{W}+\delta_{g}\right) \psi_{\mu}^{\alpha}=\xi^{\nu} \partial_{\nu} \psi_{\mu}^{\alpha}+\partial_{\mu} \xi^{\nu} \psi_{\nu}^{\alpha}-\frac{1}{4} \lambda_{a b} \gamma^{a b} \psi_{\mu}^{\alpha}+\frac{1}{2} \Lambda \psi_{\mu}^{\alpha}-\zeta^{\alpha \beta} \psi_{\mu}^{\beta}
$$

The fermionic transformations $\delta_{Q}$ and $\delta_{S}$ are given by

$$
\begin{aligned}
\delta_{Q} e_{\mu}{ }^{a}= & \frac{1}{4} \bar{\epsilon}^{\alpha} \gamma^{a} \psi_{\mu}^{\alpha}, \quad \delta_{Q} \psi_{\mu}^{\alpha}=D_{\mu} \epsilon^{\alpha}, \\
\delta_{Q} B_{\mu}^{\alpha \beta}= & -\bar{\epsilon}^{[\alpha} \psi_{\mu+}^{\beta]}+\frac{1}{2 \sqrt{2}} \bar{\epsilon}^{\gamma} \gamma_{\mu} \lambda^{\alpha \beta \gamma}-\frac{1}{2 \sqrt{2}} \bar{\epsilon}^{\gamma} \psi_{\mu}^{\delta} E^{\alpha \beta \gamma \delta}, \\
\delta_{Q} \lambda^{\alpha \beta \gamma}= & -\frac{3}{4 \sqrt{2}} \gamma^{\mu \nu} \epsilon^{[\alpha} \hat{G}_{\mu \nu}^{\beta \gamma]}+\epsilon^{\delta} D^{\alpha \beta \gamma \delta}-\frac{1}{2} \gamma^{\mu} \epsilon^{\delta} \hat{D}_{\mu} E^{\alpha \beta \gamma \delta} \\
& -\frac{3}{4 \sqrt{2}} \epsilon^{\delta} E^{\epsilon \eta[\alpha \beta} E^{\gamma \delta] \epsilon \eta}, \\
\delta_{Q} D^{\alpha_{1} \cdots \alpha_{4}}= & \bar{\epsilon}^{\left[\alpha_{1}\right.} \lambda_{+}^{\left.\alpha_{2} \alpha_{3} \alpha_{4}\right]}+\frac{1}{4 !} \eta \epsilon^{\alpha_{1} \cdots \alpha_{8}} \bar{\epsilon}^{\alpha_{5}} \lambda_{+}^{\alpha_{6} \alpha_{7} \alpha_{8}}, \\
\delta_{Q} E^{\alpha_{1} \cdots \alpha_{4}}= & \bar{\epsilon}^{\left[\alpha_{1}\right.} \lambda^{\left.\alpha_{2} \alpha_{3} \alpha_{4}\right]}-\frac{1}{4 !} \eta \epsilon^{\alpha_{1} \cdots \alpha_{8}} \bar{\epsilon}^{\alpha_{5}} \lambda^{\alpha_{6} \alpha_{7} \alpha_{8}}
\end{aligned}
$$

and

$$
\begin{aligned}
\delta_{S} e_{\mu}^{a} & =0, \quad \delta_{S} \psi_{\mu}^{\alpha}=\gamma_{\mu} \eta^{\alpha}, \quad \delta_{S} B_{\mu}^{\alpha \beta}=\frac{1}{2} \bar{\eta}^{[\alpha} \psi_{\mu}^{\beta]}, \\
\delta_{S} \lambda^{\alpha \beta \gamma} & =\eta^{\delta} E^{\alpha \beta \gamma \delta}, \quad \delta_{S} E^{\alpha_{1} \cdots \alpha_{4}}=0 \\
\delta_{S} D^{\alpha_{1} \cdots \alpha_{4}} & =-\frac{1}{2}\left(\bar{\eta}^{\left[\alpha_{1}\right.} \lambda^{\left.\alpha_{2} \alpha_{3} \alpha_{4}\right]}+\frac{1}{4 !} \eta \epsilon^{\alpha_{1} \cdots \alpha_{8}} \bar{\eta}^{\left[\alpha_{5}\right.} \lambda^{\left.\alpha_{6} \alpha_{7} \alpha_{8}\right]}\right) .
\end{aligned}
$$


Here, we have defined

$$
\begin{aligned}
\psi_{\mu+}^{\alpha}= & \frac{1}{4} \gamma^{\rho \sigma} \gamma_{\mu} \psi_{\rho \sigma}^{\alpha}, \quad \psi_{\mu \nu}^{\alpha}=D_{[\mu} \psi_{\nu]}^{\alpha}, \\
\lambda_{+}^{\alpha \beta \gamma}= & -\frac{1}{2} \gamma^{\mu} \hat{D}_{\mu} \lambda^{\alpha \beta \gamma}-\gamma^{\mu} \psi_{\mu+}^{\delta} E^{\alpha \beta \gamma \delta}+\frac{3}{2 \sqrt{2}} E^{\delta \epsilon[\alpha \beta} \lambda^{\gamma] \delta \epsilon} \\
\hat{G}_{\mu \nu}^{\alpha \beta}= & G_{\mu \nu}^{\alpha \beta}+2 \bar{\psi}_{[\mu}^{[\alpha} \psi_{\nu]+}^{\beta]}-\frac{1}{\sqrt{2}} \bar{\psi}_{[\mu}^{\gamma} \gamma_{\nu]} \lambda^{\alpha \beta \gamma}+\frac{1}{2 \sqrt{2}} \bar{\psi}_{[\mu}^{\gamma} \psi_{\nu]}^{\delta} E^{\alpha \beta \gamma \delta}, \\
\hat{D}_{\mu} E^{\alpha_{1} \cdots \alpha_{4}}= & D_{\mu} E^{\alpha_{1} \cdots \alpha_{4}}-\bar{\psi}_{\mu}^{\left[\alpha_{1}\right.} \lambda^{\left.\alpha_{2} \alpha_{3} \alpha_{4}\right]}+\frac{1}{4 !} \eta \epsilon^{\alpha_{1} \cdots \alpha_{8}} \bar{\psi}_{\mu}^{\alpha_{5}} \lambda^{\alpha_{6} \alpha_{7} \alpha 8}, \\
\hat{D}_{\mu} \lambda^{\alpha \beta \gamma}= & D_{\mu} \lambda^{\alpha \beta \gamma}+\frac{3}{4 \sqrt{2}} \gamma^{\rho \sigma} \psi_{\mu}^{[\alpha} \hat{G}_{\rho \sigma}^{\beta \gamma]}-\psi_{\mu}^{\delta} D^{\alpha \beta \gamma \delta}+\frac{1}{2} \gamma^{\rho} \psi_{\mu}^{\delta} \hat{D}_{\rho} E^{\alpha \beta \gamma \delta} \\
& +\frac{3}{4 \sqrt{2}} \psi_{\mu}^{\delta} E^{\epsilon \eta[\alpha \beta} E^{\gamma \delta] \epsilon \eta} .
\end{aligned}
$$

The covariant derivative $D_{\mu}$ contains the spin connection and the $\mathrm{SO}(8)$ gauge fields and is given by, e.g., for $\epsilon^{\alpha}$

$$
D_{\mu} \epsilon^{\alpha}=\left(\partial_{\mu}+\frac{1}{4} \hat{\omega}_{\mu a b} \gamma^{a b}\right) \epsilon^{\alpha}+B_{\mu}^{\alpha \beta} \epsilon^{\beta}
$$

The spin connection $\hat{\omega}_{\mu a b}$ satisfies the torsion condition

$$
D_{\mu} e_{\nu}^{a}-D_{\nu} e_{\mu}^{a}=\frac{1}{4} \bar{\psi}_{\mu} \gamma^{a} \psi_{\nu}
$$

and is given by

$$
\hat{\omega}_{\mu a b}=\omega_{\mu a b}(e)+\frac{1}{8}\left(\bar{\psi}_{a} \gamma_{\mu} \psi_{b}+\bar{\psi}_{\mu} \gamma_{a} \psi_{b}-\bar{\psi}_{\mu} \gamma_{b} \psi_{a}\right)
$$

where $\omega_{\mu a b}(e)$ is the spin connection without torsion. The Ricci tensor made from the spin connection $\hat{\omega}_{\mu a b}$ is not a symmetric tensor but has an antisymmetric part

$$
R_{[\mu \nu]}=-\frac{3}{4} \bar{\psi}_{[\rho} \gamma^{\rho} \psi_{\mu \nu]}
$$

$\hat{D}_{\mu}$ is the supercovariant derivative, which transforms without $\partial_{\mu} \epsilon$ terms under the local supertransformation. $\hat{D}_{\mu} E^{\alpha \beta \gamma \delta}$ satisfies the same self-duality condition as $E^{\alpha \beta \gamma \delta}$ in (3.1). The field strength of the $\mathrm{SO}(8)$ gauge fields is

$$
G_{\mu \nu}^{\alpha \beta}=\partial_{\mu} B_{\nu}^{\alpha \beta}-\partial_{\nu} B_{\mu}^{\alpha \beta}+B_{\mu}^{\alpha \gamma} B_{\nu}^{\gamma \beta}-B_{\nu}^{\alpha \gamma} B_{\mu}^{\gamma \beta}
$$

and $\hat{G}_{\mu \nu}^{\alpha \beta}$ is its supercovariantization. By linearization the transformations (3.3), (3.4) reduce to the transformations in [18, 20]. 
The above local transformations satisfy a commutator algebra which closes offshell, i.e., without using any constraints on the fields such as field equations. This is guaranteed from the fact that the local transformations of the $D=4 S O(8)$ gauged supergravity satisfy a closed commutator algebra on-shell and the fact that the field equations of the $D=4$ theory do not impose any constraint on the boundary values $\Phi_{0}=\left(e_{0 \mu}{ }^{a}, \psi_{0 \mu-}, B_{0 \mu}, \lambda_{0-}, D_{0}, E_{0}\right)$, which become the fields of the $D=3$ conformal supergravity multiplet as discussed in the previous section. Explicit forms of the commutators will be given below.

So far we have used only negative chirality spinor indices $\alpha, \beta, \cdots=1,2, \cdots, 8$ of $\mathrm{SO}(8)$. On the other hand the BLG theory contains fields with vector indices $I, J, \cdots=1,2, \cdots, 8$ and positive chirality spinor indices $\dot{\alpha}, \dot{\beta}, \cdots=1,2, \cdots, 8$ of $\mathrm{SO}(8)$. To couple the conformal supergravity to the BLG theory we need the Clebsch-Gordan coefficients which connect these three kinds of indices of $\mathrm{SO}(8)$. They are provided by the $16 \times 16$ gamma matrices of $\mathrm{SO}(8)$

$$
\Gamma^{I}=\left(\begin{array}{cc}
0 & \Sigma^{I} \\
\bar{\Sigma}^{I} & 0
\end{array}\right)
$$

where $\Sigma^{I}$ and $\bar{\Sigma}^{I}=\left(\Sigma^{I}\right)^{T}$ are $8 \times 8$ matrices with components $\left(\Sigma^{I}\right)^{\alpha \dot{\beta}}$ and $\left(\bar{\Sigma}^{I}\right)^{\dot{\alpha} \beta}$. These $\Sigma$-matrices satisfy identities given in Appendix B.

To couple the conformal supergravity to the BLG theory it is convenient to define fields with vector and positive chirality spinor indices instead of negative chirality spinor indices. For that purpose we introduce combinations of $\Sigma$-matrices*

$$
\begin{aligned}
t_{I J}^{\alpha \beta \gamma \delta} & =\left(\Sigma_{K I}\right)^{[\alpha \beta}\left(\Sigma_{K J}\right)^{\gamma \delta]} \\
t_{I J K L}^{\alpha \beta \gamma \delta} & =\left(\Sigma_{[I J}\right)^{[\alpha \beta}\left(\Sigma_{K L]}\right)^{\gamma \delta]} \\
u_{I \dot{\alpha}}^{\alpha \beta \gamma} & =-\left(\Sigma_{I J}\right)^{[\alpha \beta}\left(\Sigma_{J}\right)^{\gamma] \dot{\alpha}}
\end{aligned}
$$

where $\Sigma^{I J}=\Sigma^{[I} \bar{\Sigma}^{J]}$, and upper and lower indices are not distinguished. They satisfy

$$
\begin{gathered}
t_{I J}^{\alpha_{1} \cdots \alpha_{4}}=\frac{1}{4 !} \epsilon^{\alpha_{1} \cdots \alpha_{8}} t_{I J}^{\alpha_{5} \cdots \alpha_{8}}, \quad t_{I J K L}^{\alpha_{1} \cdots \alpha_{4}}=-\frac{1}{4 !} \epsilon^{\alpha_{1} \cdots \alpha_{8}} t_{I J K L}^{\alpha_{5} \cdots \alpha_{8}}, \\
t_{I J}^{\alpha \beta \gamma \delta}=t_{J I}^{\alpha \beta \gamma \delta}, \quad t_{I I}^{\alpha \beta \gamma \delta}=0, \quad t_{I_{1} \cdots I_{4}}^{\alpha \beta \gamma \delta}=\frac{1}{4 !} \epsilon^{I_{1} \cdots I_{8}} t_{I_{5} \cdots I_{8}}^{\alpha \beta \gamma \delta}, \quad\left(\Sigma^{I}\right)^{\alpha \dot{\beta}} u_{I \dot{\beta}}^{\beta \gamma \delta}=0 .
\end{gathered}
$$

*These were used in [24] in a study of the superstring field theory in the light-cone gauge. See Appendix A of [24] for further identities which they satisfy. 
Note that $t_{I J}^{\alpha_{1} \cdots \alpha_{4}}$ is self-dual and $t_{I J K L}^{\alpha_{1} \cdots \alpha_{4}}$ is anti self-dual in the four spinor indices. We can define new $\mathrm{SO}(8)$ gauge fields $B_{\mu}^{I J}$ and spinor fields $\lambda^{I \dot{\alpha}}$ by

$$
\begin{aligned}
B_{\mu}^{I J} & =\frac{1}{4}\left(\Sigma^{I J}\right)^{\alpha \beta} B_{\mu}^{\alpha \beta}, & B_{\mu}^{\alpha \beta} & =\frac{1}{4}\left(\Sigma^{I J}\right)^{\alpha \beta} B_{\mu}^{I J}, \\
\lambda^{I \dot{\alpha}} & =\frac{1}{48} u_{I \dot{\alpha}}^{\alpha \beta \gamma} \lambda^{\alpha \beta \gamma}, & \lambda^{\alpha \beta \gamma} & =u_{I \dot{\alpha}}^{\alpha \beta \gamma} \lambda^{I \dot{\alpha}},
\end{aligned}
$$

which satisfy

$$
B_{\mu}^{I J}=-B_{\mu}^{J I}, \quad\left(\Sigma^{I}\right)^{\alpha \dot{\beta}} \lambda^{I \dot{\beta}}=0 .
$$

The field strength of the new gauge field is

$$
G_{\mu \nu}^{I J}=\partial_{\mu} B_{\nu}^{I J}-\partial_{\nu} B_{\mu}^{I J}+B_{\mu}^{I K} B_{\nu}^{K J}-B_{\nu}^{I K} B_{\mu}^{K J}
$$

Definitions of new scalar fields depend on the self-duality parameter $\eta$ in (3.1). When the parameter is $\eta=+1, D^{\alpha \beta \gamma \delta}$ is self-dual and $E^{\alpha \beta \gamma \delta}$ is anti self-dual. Then, we can define the scalar fields $D^{I J}$ and $E^{I J K L}$ by

$$
\begin{aligned}
D^{I J} & =\frac{1}{4 \cdot 4 !} t_{I J}^{\alpha \beta \gamma \delta} D^{\alpha \beta \gamma \delta}, & D^{\alpha \beta \gamma \delta} & =\frac{1}{4} t_{I J}^{\alpha \beta \gamma \delta} D^{I J}, \\
E^{I J K L} & =\frac{1}{256} t_{I J K L}^{\alpha \beta \gamma \delta} E^{\alpha \beta \gamma \delta}, & E^{\alpha \beta \gamma \delta} & =t_{I J K L}^{\alpha \beta \gamma \delta} E^{I J K L},
\end{aligned}
$$

which satisfy

$$
D^{I J}=D^{J I}, \quad D^{I I}=0, \quad E^{I J K L}=\frac{1}{4 !} \epsilon^{I J K L M N P Q} E^{M N P Q} .
$$

(One can further define $E^{\dot{\alpha} \dot{\beta}}=\left(\bar{\Sigma}^{I J K L}\right)^{\dot{\alpha} \dot{\beta}} E^{I J K L}$, which is symmetric and traceless in $(\dot{\alpha} \dot{\beta})$, but we use $E^{I J K L}$ in the following.) On the other hand, when the parameter is $\eta=-1$, we can define $D^{I J K L}$ and $E^{I J}$ as in (3.17), (3.18) with $D$ and $E$ interchanged. Note that the two cases $\eta= \pm 1$ are not equivalent since $D$ and $E$ have different Weyl weights. In the following we rewrite the fermionic transformations in terms of the new fields. 


\section{$3.1 \eta=+1$}

In this case the fermionic transformations $\delta_{Q}$ and $\delta_{S}$ in (3.3) and (3.4) become

$$
\begin{aligned}
\delta_{Q} e_{\mu}{ }^{a}= & \frac{1}{4} \bar{\epsilon} \gamma^{a} \psi_{\mu}, \quad \delta_{Q} \psi_{\mu}=D_{\mu} \epsilon, \\
\delta_{Q} B_{\mu}^{I J}= & -\frac{1}{4} \bar{\epsilon} \Sigma^{I J} \psi_{\mu+}+\sqrt{2} \bar{\epsilon} \gamma_{\mu} \Sigma^{[I} \lambda^{J]}-\sqrt{2} \bar{\epsilon} \Sigma^{K L} \psi_{\mu} E^{I J K L}, \\
\delta_{Q} \lambda^{I}= & -\frac{1}{128 \sqrt{2}} \gamma^{\mu \nu}\left(\bar{\Sigma}^{I K L}-6 \delta^{I K} \bar{\Sigma}^{L}\right) \epsilon \hat{G}_{\mu \nu}^{K L}+\frac{1}{4} \bar{\Sigma}^{J} \epsilon D^{I J} \\
& +\frac{1}{6} \gamma^{\mu} \bar{\Sigma}^{J K L} \epsilon \hat{D}_{\mu} E^{I J K L}+2 \sqrt{2} \bar{\Sigma}^{J K L} \epsilon E^{M N[I J} E^{K L] M N}, \\
\delta_{Q} D^{I J}= & -\bar{\epsilon} \Sigma^{(I} \lambda_{+}^{J)}, \quad \delta_{Q} E^{I J K L}=\frac{1}{8} \bar{\epsilon} \Sigma^{[I J K} \lambda^{L]}
\end{aligned}
$$

and

$$
\begin{aligned}
& \delta_{S} e_{\mu}{ }^{a}=0, \quad \delta_{S} \psi_{\mu}=\gamma_{\mu} \eta, \quad \delta_{S} B_{\mu}^{I J}=\frac{1}{8} \bar{\eta} \Sigma^{I J} \psi_{\mu}, \\
& \delta_{S} \lambda^{I}=-\frac{1}{3} \bar{\Sigma}^{J K L} \eta E^{I J K L}, \quad \delta_{S} D^{I J}=\frac{1}{2} \bar{\eta} \Sigma^{(I} \lambda^{J)}, \quad \delta_{S} E^{I J K L}=0,
\end{aligned}
$$

where

$$
\lambda_{+}^{I}=-\frac{1}{2} \gamma^{\mu} \hat{D}_{\mu} \lambda^{I}+\frac{1}{3} \gamma^{\mu} \bar{\Sigma}^{J K L} \psi_{\mu+} E^{I J K L}+\frac{1}{\sqrt{2}}\left(\bar{\Sigma}^{I J K L}-5 \delta^{I J} \bar{\Sigma}^{K L}\right) E^{J K L M} \lambda^{M} .
$$

We have suppressed $\mathrm{SO}(8)$ spinor indices $\alpha, \dot{\alpha}, \cdots$. Explicit forms of the supercovariant quantities $\hat{G}_{\mu \nu}^{I J}, \hat{D}_{\mu} E^{I J K L}, \hat{D}_{\mu} \lambda^{I}$ in terms of the new fields can be found from (3.5) or more easily from the supertransformations (3.19). $\lambda_{+}^{I}$ in (3.21) is related to $\lambda_{+}^{\alpha \beta \gamma}$ in (3.5) as $\lambda_{+}^{I \dot{\alpha}}=\frac{1}{48} u_{I \dot{\alpha}}^{\alpha \beta \gamma} \lambda_{+}^{\alpha \beta \gamma}$ and satisfies $\Sigma^{I} \lambda_{+}^{I}=0$.

As explained above the commutator algebra of the local transformations closes off-shell. Indeed, by lengthy but straightforward calculations using $\mathrm{SO}(8)$ identities in Appendix B we found the off-shell commutation relations as

$$
\begin{aligned}
{\left[\delta_{Q}\left(\epsilon_{1}\right), \delta_{Q}\left(\epsilon_{2}\right)\right] } & =\delta_{G}(\xi)+\delta_{L}(\lambda)+\delta_{g}(\zeta)+\delta_{Q}\left(\epsilon^{\prime}\right)+\delta_{S}\left(\eta^{\prime}\right), \\
{\left[\delta_{Q}(\epsilon), \delta_{S}(\eta)\right] } & =\delta_{W}(\Lambda)+\delta_{L}\left(\lambda^{\prime}\right)+\delta_{g}\left(\zeta^{\prime}\right)+\delta_{S}\left(\eta^{\prime \prime}\right), \\
{\left[\delta_{S}\left(\eta_{1}\right), \delta_{S}\left(\eta_{2}\right)\right] } & =0
\end{aligned}
$$


where the transformation parameters on the right-hand sides are

$$
\begin{aligned}
\xi^{\mu}= & \frac{1}{4} \bar{\epsilon}_{2} \gamma^{\mu} \epsilon_{1}, \quad \lambda_{a b}=-\xi^{\mu} \hat{\omega}_{\mu a b}, \\
\zeta^{I J}= & -\xi^{\mu} B_{\mu}^{I J}-\sqrt{2} \bar{\epsilon}_{2} \Sigma^{K L} \epsilon_{1} E^{I J K L}, \quad \epsilon^{\prime}=-\xi^{\mu} \psi_{\mu}, \\
\eta^{\prime}= & \frac{1}{32} \xi^{\mu}\left(14 \gamma^{\rho \sigma} \gamma_{\mu}-\gamma_{\mu} \gamma^{\rho \sigma}\right) \psi_{\rho \sigma}-\left(\frac{1}{256} \Sigma^{I J} \gamma^{\rho \sigma} \psi_{\rho \sigma}+\frac{1}{2 \sqrt{2}} \Sigma^{I} \lambda^{J}\right) \bar{\epsilon}_{2} \Sigma^{I J} \epsilon_{1} \\
& -\frac{1}{256 \cdot 4 !} \Sigma^{I J K L}\left(2 \gamma^{\rho \sigma} \gamma_{\mu}+\gamma_{\mu} \gamma^{\rho \sigma}\right) \psi_{\rho \sigma} \bar{\epsilon}_{2} \gamma^{\mu} \Sigma^{I J K L} \epsilon_{1}, \\
\Lambda= & -\frac{1}{4} \bar{\epsilon} \eta, \quad \lambda_{a b}^{\prime}=\frac{1}{4} \bar{\epsilon} \gamma_{a b} \eta, \quad \zeta^{I J}=-\frac{1}{8} \bar{\epsilon} \Sigma^{I J} \eta, \quad \eta^{\prime \prime}=\frac{1}{8} \gamma^{\mu} \epsilon \bar{\eta} \psi_{\mu} .
\end{aligned}
$$

The $\mathrm{SO}(8)$ parameter $\zeta^{I J}$ is related to $\zeta^{\alpha \beta}$ as $\zeta^{I J}=\frac{1}{4}\left(\Sigma^{I J}\right)^{\alpha \beta} \zeta^{\alpha \beta}$. We have explicitly checked these commutation relations on all the fields except for $\left[\delta_{Q}, \delta_{Q}\right] \lambda^{I}$ and $\left[\delta_{Q}, \delta_{Q}\right] D^{I J}$, which are the hardest to calculate.

\section{$3.2 \eta=-1$}

In this case the fermionic transformations $\delta_{Q}$ and $\delta_{S}$ in (3.3) and (3.4) become

$$
\begin{aligned}
\delta_{Q} e_{\mu}{ }^{a}= & \frac{1}{4} \bar{\epsilon} \gamma^{a} \psi_{\mu}, \quad \delta_{Q} \psi_{\mu}=D_{\mu} \epsilon \\
\delta_{Q} B_{\mu}^{I J}= & -\frac{1}{4} \bar{\epsilon} \Sigma^{I J} \psi_{\mu+}+\sqrt{2} \bar{\epsilon} \gamma_{\mu} \Sigma^{[I} \lambda^{J]}+\frac{1}{2 \sqrt{2}} \bar{\epsilon} \Sigma^{K[I} \psi_{\mu} E^{J] K}, \\
\delta_{Q} \lambda^{I}= & -\frac{1}{128 \sqrt{2}} \gamma^{\mu \nu}\left(\overline{\Sigma^{I K L}}-6 \delta^{I K} \bar{\Sigma}{ }^{L}\right) \epsilon \hat{G}_{\mu \nu}^{K L}-\frac{1}{3} \bar{\Sigma}^{J K L} \epsilon D^{I J K L} \\
& -\frac{1}{8} \gamma^{\mu} \bar{\Sigma}^{J} \epsilon \hat{D}_{\mu} E^{I J}-\frac{1}{4 \sqrt{2}} \bar{\Sigma}^{J} \epsilon\left(E^{I K} E^{J K}-\frac{1}{8} \delta^{I J} E^{K L} E^{K L}\right), \\
\delta_{Q} D^{I J K L}= & \frac{1}{8} \bar{\epsilon} \Sigma^{[I J K} \lambda_{+}^{L]}, \quad \delta_{Q} E^{I J}=-\bar{\epsilon} \Sigma^{(I} \lambda^{J)}
\end{aligned}
$$

and

$$
\begin{aligned}
\delta_{S} e_{\mu}{ }^{a} & =0, \quad \delta_{S} \psi_{\mu}=\gamma_{\mu} \eta, \quad \delta_{S} B_{\mu}^{I J}=\frac{1}{8} \bar{\eta} \Sigma^{I J} \psi_{\mu}, \\
\delta_{S} \lambda^{I} & =\frac{1}{4} \bar{\Sigma}^{J} \eta E^{I J}, \quad \delta_{S} D^{I J K L}=-\frac{1}{16} \bar{\eta} \Sigma^{[I J K} \lambda^{L]}, \quad \delta_{S} E^{I J}=0,
\end{aligned}
$$

where

$$
\lambda_{+}^{I}=-\frac{1}{2} \gamma^{\mu} \hat{D}_{\mu} \lambda^{I}-\frac{1}{4} \gamma^{\mu} \bar{\Sigma}^{J} \psi_{\mu+} E^{I J}-\frac{1}{4 \sqrt{2}}\left(\bar{\Sigma}^{I K}-7 \delta^{I K}\right) \lambda^{L} E^{K L} .
$$




\begin{tabular}{|c|c|c|c|}
\hline Field & $X_{i}^{I}$ & $\Psi_{i}^{\dot{\alpha}}$ & $\tilde{A}_{\mu}{ }^{j}{ }_{i}$ \\
\hline Weyl weight & $-\frac{1}{2}$ & -1 & 0 \\
\hline $\mathrm{SO}(8)$ representation & $\mathbf{8}_{v}$ & $\mathbf{8}_{c}$ & $\mathbf{1}$ \\
\hline
\end{tabular}

Table 2: The field content of the BLG multiplet.

The commutators of the fermionic transformations are given by (3.22). The parameters on the right-hand sides are given by (3.23) except for

$$
\zeta^{I J}=-\xi^{\mu} B_{\mu}^{I J}+\frac{1}{2 \sqrt{2}} \bar{\epsilon}_{2} \Sigma^{K[I} \epsilon_{1} E^{J] K} .
$$

\section{The BLG theory coupled to conformal supergravity}

The BLG theory [1 3] is a three-dimensional field theory invariant under $\mathcal{N}=8$ superconformal transformations. It is based on an algebraic structure called a 3algebra with a four-index structure constant $f^{k l j}{ }_{i}$. The field content of the theory is real scalar fields $X_{i}^{I}(x)$, Majorana spinor fields $\Psi_{i}^{\dot{\alpha}}(x)$ and Chern-Simons gauge fields $\tilde{A}_{\mu}{ }^{j} i(x)=A_{\mu k l}(x) f^{k l j}{ }_{i}\left(A_{\mu k l}=-A_{\mu l k}\right)$ as shown in Table 2. Here, $I, J, \cdots=$ $1,2, \cdots, 8$ and $\dot{\alpha}, \dot{\beta}, \cdots=1,2, \cdots, 8$ are vector indices and positive chirality spinor indices of $\mathrm{SO}(8)$ respectively as in the previous section, and $i, j, \cdots=1,2, \cdots, n$ are indices of the 3 -algebra. The 3 -algebra indices are raised and lowered by a constant metric $h^{i j}, h_{i j}$. The structure constant of the 3-algebra $f^{i j k}{ }_{l}$ satisfies

$$
f^{i j k l}=f^{[i j k l]}, \quad f_{p}^{m n[i} f^{j k l] p}=0 .
$$

The 3-algebra gauge transformations of the fields are

$$
\begin{aligned}
\delta_{g 3} X_{i}^{I} & =X_{j}^{I} \tilde{\Lambda}^{j}{ }_{i}, \quad \delta_{g 3} \Psi_{i}=\Psi_{j} \tilde{\Lambda}^{j}{ }_{i}, \\
\delta_{g 3} \tilde{A}_{\mu}{ }_{i}{ }_{i} & =D_{\mu} \tilde{\Lambda}^{j}{ }_{i}=\partial_{\mu} \tilde{\Lambda}^{j}{ }_{i}-\tilde{\Lambda}^{j}{ }_{k} \tilde{A}_{\mu}{ }^{k}{ }_{i}+\tilde{A}_{\mu}{ }_{k}{ }_{k} \tilde{\Lambda}^{k}{ }_{i},
\end{aligned}
$$

where $\tilde{\Lambda}^{j}{ }_{i}(x)=\Lambda_{k l}(x) f^{k l j}{ }_{i}$ is a transformation parameter.

We would like to couple the BLG theory to the off-shell conformal supergravity constructed in the previous sections. We will only consider the $\eta=+1$ case since we 
find difficulties in coupling the $\eta=-1$ conformal supergravity to the BLG theory as will be discussed in section 6 . By the standard Noether procedure starting from the theory in a flat background [1-3] we can obtain local transformation laws of the fields and a Lagrangian in the conformal supergravity background.

First we shall give local transformation laws of the BLG fields. Transformations of the conformal supergravity fields remain the same as in the previous section since they have the closed commutator algebra off-shell. Weyl weights and $\mathrm{SO}(8)$ representations of the fields are given in Table 2. Bosonic transformation laws other than the Weyl transformation are obvious from the index structure of the fields. Local supertransformations $\delta_{Q}$ and super Weyl transformations $\delta_{S}$ of the BLG fields are given by

$$
\begin{aligned}
\delta_{Q} X_{i}^{I} & =\frac{1}{2 \sqrt{2}} \bar{\epsilon} \Sigma^{I} \Psi_{i} \\
\delta_{Q} \Psi_{i} & =\frac{1}{2 \sqrt{2}} \gamma^{\mu} \bar{\Sigma}^{I} \epsilon \hat{D}_{\mu} X_{i}^{I}+\frac{1}{12 \sqrt{2}} f_{i}^{j k l} X_{j}^{J} X_{k}^{K} X_{l}^{L} \bar{\Sigma}^{J K L} \epsilon-\frac{2}{3} \bar{\Sigma}^{J K L} \epsilon E^{I J K L} X_{i}^{I}, \\
\delta_{Q} \tilde{A}_{\mu i}^{j} & =\frac{1}{2 \sqrt{2}} \bar{\epsilon} \gamma_{\mu} \Sigma^{I} \Psi_{k} X_{l}^{I} f^{k l j}{ }_{i}-\frac{1}{8} \bar{\epsilon} \Sigma^{K L} \psi_{\mu} X_{k}^{K} X_{l}^{L} f^{k l j}{ }_{i}
\end{aligned}
$$

and

$$
\delta_{S} X_{i}^{I}=0, \quad \delta_{S} \Psi_{i}=-\frac{1}{2 \sqrt{2}} \bar{\Sigma}^{I} \eta X_{i}^{I}, \quad \delta_{S} \tilde{A}_{\mu i}^{j}=0
$$

Here, $\hat{D}_{\mu}$ is a supercovariant derivative

$$
\hat{D}_{\mu} X_{i}^{I}=D_{\mu} X_{i}^{I}-\frac{1}{2 \sqrt{2}} \bar{\psi}_{\mu} \Sigma^{I} \Psi_{i}
$$

The covariant derivative $D_{\mu}$ contains the 3 -algebra gauge fields in addition to the spin connection and the $\mathrm{SO}(8)$ gauge fields:

$$
\begin{aligned}
D_{\mu} X_{i}^{I} & =\partial_{\mu} X_{i}^{I}+B_{\mu}^{I J} X_{i}^{J}-X_{j}^{I} \tilde{A}_{\mu}{ }^{j}{ }_{i}, \\
D_{\mu} \Psi_{i} & =\left(\partial_{\mu}+\frac{1}{4} \hat{\omega}_{\mu a b} \gamma^{a b}+\frac{1}{4} B_{\mu}^{I J} \bar{\Sigma}^{I J}\right) \Psi_{i}-\Psi_{j} \tilde{A}_{\mu}{ }^{j}{ }_{i} .
\end{aligned}
$$


The Lagrangian of the BLG theory coupled to the conformal supergravity background can be written as

$$
\begin{aligned}
\mathcal{L}= & \mathcal{L}_{0}+\mathcal{L}_{1}+\mathcal{L}_{2}, \\
\mathcal{L}_{0}= & -\frac{1}{2} e D_{\mu} X^{i I} D^{\mu} X_{i}^{I}-\frac{1}{2} e \bar{\Psi}^{i} \gamma^{\mu} D_{\mu} \Psi_{i}+\frac{1}{4} e f^{i j k l} \bar{\Psi}_{i} \bar{\Sigma}^{I J} \Psi_{j} X_{k}^{I} X_{l}^{J}-e V \\
& +\frac{1}{2} \epsilon^{\mu \nu \rho}\left(f^{i j k l} A_{\mu i j} \partial_{\nu} A_{\rho k l}+\frac{2}{3} f_{p}^{k l i} f^{m n p j} A_{\mu i j} A_{\nu k l} A_{\rho m n}\right) \\
\mathcal{L}_{1}= & \frac{1}{2 \sqrt{2}} e \bar{\psi}_{\mu} \gamma^{\nu} \gamma^{\mu} \Sigma^{I} \Psi^{i} D_{\nu} X_{i}^{I}+\frac{1}{12 \sqrt{2}} e \bar{\psi}_{\mu} \gamma^{\mu} \Sigma^{J K L} \Psi_{i} f^{i j k l} X_{j}^{J} X_{k}^{K} X_{l}^{L} \\
& -\frac{1}{16} e \bar{\psi}_{\mu} \gamma^{\mu \nu \rho} \Sigma^{I J} \psi_{\nu} X^{i I} D_{\rho} X_{i}^{J}-\frac{1}{4 \sqrt{2}} e \bar{\Psi}^{i} \gamma^{\mu \nu} \bar{\Sigma}^{I} D_{\mu} \psi_{\nu} X_{i}^{I}-\frac{1}{16} e R X^{2} \\
& +\frac{1}{32} e \bar{\psi}_{\mu} \gamma^{\mu \nu \rho} D_{\nu} \psi_{\rho} X^{2}-\frac{1}{192} e \bar{\psi}_{\mu} \gamma^{\mu \nu} \Sigma^{I J K L} \psi_{\nu} f^{i j k l} X_{i}^{I} X_{j}^{J} X_{k}^{K} X_{l}^{L}, \\
\mathcal{L}_{2}= & -4 e \bar{\lambda}^{I} \Psi^{i} X_{i}^{I}+\frac{8}{3 \sqrt{2}^{2}} e E^{I J K L} f^{i j k l} X_{i}^{I} X_{j}^{J} X_{k}^{K} X_{l}^{L} \\
& -\frac{2}{3} e \bar{\psi}_{\mu} \gamma^{\mu} \Sigma^{J K L} \Psi^{i} E^{I J K L} X_{i}^{I}+\sqrt{2}_{2} D^{I J} X^{i I} X_{i}^{J} \\
& +\frac{1}{\sqrt{2}} e \bar{\psi}_{\mu} \gamma^{\mu} \Sigma^{I} \lambda^{J} X^{i I} X_{i}^{J}+\frac{1}{6 \sqrt{2}^{2}} e \bar{\psi}_{\mu} \gamma^{\mu \nu} \Sigma^{I K L M} \psi_{\nu} E^{J K L M} X^{i I} X_{i}^{J} \\
& -\frac{4}{3} e E^{I J K L} E^{I J K L} X^{2}+\frac{1}{6 \sqrt{2}} e E^{I J K L} \bar{\Psi}^{i} \bar{\Sigma}^{I J K L} \Psi_{i} \\
& +\frac{1}{32} e \bar{\psi}_{\mu} \gamma^{\nu} \gamma^{\mu} \psi_{\nu} \bar{\Psi}^{i} \Psi_{i}+\frac{1}{128} e \bar{\psi}_{\mu}\left(\gamma^{\mu \nu}{ }_{\rho}-g^{\mu \nu} \gamma_{\rho}\right) \Sigma^{I J} \psi_{\nu} \bar{\Psi}^{i} \gamma^{\rho} \bar{\Sigma}^{I J} \Psi_{i} .
\end{aligned}
$$

Here, $R$ is the scalar curvature made from the spin connection $\hat{\omega}_{\mu a b}$ in (3.8), and we have defined

$$
V=\frac{1}{12} f^{i j k l} f_{l}^{m n p} X_{i}^{I} X_{j}^{J} X_{k}^{K} X_{m}^{I} X_{n}^{J} X_{p}^{K}, \quad X^{2}=X_{i}^{I} X^{i I} .
$$

$\mathcal{L}_{0}$ is an obvious generalization of the BLG Lagrangian to the one in a curved background with the spin connection (3.8). $\mathcal{L}_{1}$ consists of couplings to $e_{\mu}{ }^{a}, \psi_{\mu}$ and $B_{\mu}^{I J}$ of the conformal supergravity multiplet. These couplings appeared also in the Lagrangian in [16, 17]. $\mathcal{L}_{2}$ contains couplings to other fields $\lambda^{I}, D^{I J}$ and $E^{I J K L}$ of the conformal supergravity multiplet, and four-Fermi terms. The Lagrangian (4.7) is invariant under all the local transformations up to a total divergence.

We found the fermionic transformations (4.3), (4.4) and the Lagrangian (4.7) by adding all possible terms which are invariant under the bosonic transformations with unknown coefficients and then fixing those coefficients such that the Lagrangian 
is invariant under the fermionic transformations. Finally, the complete invariance of the Lagrangian was shown. To show cancellations of terms cubic and higher in fermionic fields in $\delta_{Q} \mathcal{L}$ and $\delta_{S} \mathcal{L}$ we have used the identities

$$
\begin{aligned}
& \gamma_{a} \Sigma^{I J K L} \psi_{[\rho} \bar{\psi}_{\mu} \gamma^{a} \psi_{\nu]}+\gamma_{a} \psi_{[\rho} \bar{\psi}_{\mu} \gamma^{a} \Sigma^{I J K L} \psi_{\nu]}-6 \Sigma^{[I J} \psi_{[\rho} \bar{\psi}_{\mu} \Sigma^{K L]} \psi_{\nu]}=0 \text {, } \\
& \gamma^{a} \Sigma^{I J K L} \psi_{[\rho} \bar{\psi}_{\mu} \Sigma^{K L} \psi_{\nu]}+\Sigma^{K L} \psi_{[\rho} \bar{\psi}_{\mu} \gamma^{a} \Sigma^{I J K L} \psi_{\nu]} \\
& +6 \gamma^{a} \psi_{[\rho} \bar{\psi}_{\mu} \Sigma^{I J} \psi_{\nu]}+6 \Sigma^{I J} \psi_{[\rho} \bar{\psi}_{\mu} \gamma^{a} \psi_{\nu]}=0 \\
& \Sigma^{I J} \psi_{[\rho} \bar{\psi}_{\mu} \Sigma^{I J} \psi_{\nu]}+8 \gamma_{a} \psi_{[\rho} \bar{\psi}_{\mu} \gamma^{a} \psi_{\nu]}=0 \\
& \gamma_{a} \Sigma^{I J K L} \psi_{[\rho} \bar{\psi}_{\mu} \gamma^{a} \Sigma^{I J K L} \psi_{\nu]}+240 \gamma_{a} \psi_{[\rho} \bar{\psi}_{\mu} \gamma^{a} \psi_{\nu]}=0 \text {, } \\
& \Sigma^{K(I} \psi_{[\rho} \bar{\psi}_{\mu} \Sigma^{J) K} \psi_{\nu]}=\delta^{I J} \gamma_{a} \psi_{[\rho} \bar{\psi}_{\mu} \gamma^{a} \psi_{\nu]} \text {, }
\end{aligned}
$$

which can be proved by using the Fierz identities given in Appendix B.

The commutators of the local transformations now become

$$
\begin{aligned}
{\left[\delta_{Q}\left(\epsilon_{1}\right), \delta_{Q}\left(\epsilon_{2}\right)\right] } & =\delta_{G}(\xi)+\delta_{L}(\lambda)+\delta_{g}(\zeta)+\delta_{g 3}(\tilde{\Lambda})+\delta_{Q}\left(\epsilon^{\prime}\right)+\delta_{S}\left(\eta^{\prime}\right) \\
{\left[\delta_{Q}(\epsilon), \delta_{S}(\eta)\right] } & =\delta_{W}(\Lambda)+\delta_{L}\left(\lambda^{\prime}\right)+\delta_{g}\left(\zeta^{\prime}\right)+\delta_{S}\left(\eta^{\prime \prime}\right) \\
{\left[\delta_{S}\left(\eta_{1}\right), \delta_{S}\left(\eta_{2}\right)\right] } & =0
\end{aligned}
$$

The transformation parameters on the right-hand sides are given by (3.23) and

$$
\tilde{\Lambda}_{i}^{j}=-\xi^{\mu} \tilde{A}_{\mu}{ }^{j}{ }_{i}-\frac{1}{8} \bar{\epsilon}_{2} \Sigma^{K L} \epsilon_{1} X_{k}^{K} X_{l}^{L} f^{k l j}{ }_{i}
$$

for the 3-algebra gauge transformation. To show these commutation relations one has to use the field equations of the BLG fields $\Psi_{i}$ and $\tilde{A}_{\mu}{ }^{j}{ }_{i}$ derived from the Lagrangian (4.7). Thus the algebra closes only on-shell on the BLG fields as in the original BLG theory in a flat background.

\section{Supercurrent multiplet of the BLG theory}

As an application of the results in the previous sections let us obtain a supercurrent multiplet of the BLG theory in a flat background. The supercurrent multiplet is a supermultiplet which contains the energy-momentum tensor and the supercurrent in addition to other quantities [25, 14]. 
We consider the BLG theory in a flat conformal supergravity background

$$
e_{\mu}^{a}=\delta_{\mu}^{a}, \quad \psi_{\mu}=B_{\mu}^{I J}=\lambda^{I}=D^{I J}=E^{I J K L}=0 .
$$

This background is preserved by a part of the local transformations discussed in section 3. Such transformations form a supergroup $\operatorname{OSp}(8 \mid 4)$, which contains $\operatorname{SO}(2,3)$ $\times \mathrm{SO}(8)$ as a bosonic subgroup. The Lagrangian (4.7) in this background is invariant under $\operatorname{OSp}(8 \mid 4)$ transformations [26]. $\mathrm{OSp}(8 \mid 4)$ consists of conformal transformation $\delta_{C}$, superconformal transformation $\delta_{S C}$ and global $\mathrm{SO}(8)$ transformation $\delta_{\mathrm{SO}(8)}$ given by

$$
\begin{aligned}
\delta_{C}(\xi) & =\delta_{G}(\xi)+\delta_{L}(\lambda)+\delta_{W}(\Lambda), \quad \lambda_{\mu \nu}=-\partial_{[\mu} \xi_{\nu]}, \quad \Lambda=-\frac{1}{3} \partial_{\mu} \xi^{\mu}, \\
\delta_{S C}(\epsilon) & =\delta_{Q}(\epsilon)+\delta_{S}(\eta), \quad \eta=-\frac{1}{3} \gamma^{\mu} \partial_{\mu} \epsilon, \\
\delta_{\mathrm{SO}(8)}(\zeta) & =\delta_{g}(\zeta),
\end{aligned}
$$

where the transformation parameters $\xi^{\mu}, \epsilon$ and $\zeta^{I J}$ satisfy

$$
\partial_{\mu} \xi_{\nu}+\partial_{\nu} \xi_{\mu}=\frac{2}{3} \eta_{\mu \nu} \partial_{\rho} \xi^{\rho}, \quad\left(\partial_{\mu}-\frac{1}{3} \gamma_{\mu} \gamma^{\nu} \partial_{\nu}\right) \epsilon=0, \quad \partial_{\mu} \zeta^{I J}=0 .
$$

Solutions of the first two equations are conformal Killing vectors and conformal Killing spinors, respectively. The general form of conformal Killing spinors is

$$
\epsilon=\alpha+x^{\mu} \gamma_{\mu} \beta,
$$

where $\alpha$ and $\beta$ are arbitrary constant spinors.

The supercurrent multiplet of the BLG theory can be obtained by computing derivatives of the Lagrangian (4.7) with respect to the conformal supergravity fields 
and taking the flat background (5.1). We find the supercurrent multiplet as

$$
\begin{aligned}
T_{\mu \nu}= & D_{\mu} X^{i I} D_{\nu} X_{i}^{I}-\frac{1}{2} \eta_{\mu \nu} D_{\rho} X^{i I} D^{\rho} X_{i}^{I}-\frac{1}{8}\left(\partial_{\mu} \partial_{\nu}-\eta_{\mu \nu} \partial^{2}\right)\left(X^{i I} X_{i}^{I}\right) \\
& +\frac{1}{2} \bar{\Psi}^{i} \gamma_{(\mu} D_{\nu)} \Psi_{i}-\frac{1}{12} \eta_{\mu \nu} f^{i j k l} f^{m n p}{ }_{l} X_{i}^{I} X_{j}^{J} X_{k}^{K} X_{m}^{I} X_{n}^{J} X_{p}^{K} \\
S^{\mu}= & \frac{1}{2 \sqrt{2}} \gamma^{\nu} \gamma^{\mu} \Sigma^{I} \Psi^{i} D_{\nu} X_{i}^{I}+\frac{1}{4 \sqrt{2}} \partial_{\nu}\left(\gamma^{\mu \nu} \Sigma^{I} \Psi^{i} X_{i}^{I}\right) \\
& +\frac{1}{12 \sqrt{2}} f^{i j k l} \gamma^{\mu} \Sigma^{J K L} \Psi_{i} X_{j}^{J} X_{k}^{K} X_{l}^{L}, \\
J_{\mu}^{I J}= & X^{i[I} D_{\mu} X_{i}^{J]}-\frac{1}{8} \bar{\Psi}^{i} \gamma_{\mu} \bar{\Sigma}^{I J} \Psi_{i} \\
R^{I}= & \Psi^{i} X_{i}^{I}-\frac{1}{8} \bar{\Sigma}^{I} \Sigma^{J} \Psi^{i} X_{i}^{J}, \\
M^{I J}= & X_{i}^{I} X^{i J}-\frac{1}{8} \delta^{I J} X_{i}^{K} X^{i K}, \\
N^{I J K L}= & \frac{1}{2} f^{i j k l}\left(X_{i}^{I} X_{j}^{J} X_{k}^{K} X_{l}^{L}+\frac{1}{4 !} \epsilon^{I J K L M N P Q} X_{i}^{M} X_{j}^{N} X_{k}^{P} X_{l}^{Q}\right)+\frac{1}{16} \bar{\Psi}^{i} \bar{\Sigma}^{I J K L} \Psi_{i},
\end{aligned}
$$

where the covariant derivative $D_{\mu}$ contains only the 3 -algebra gauge field $\tilde{A}_{\mu}{ }_{i}{ }_{i} . T_{\mu \nu}$, $S^{\mu}$ and $J_{\mu}^{I J}$ are the energy-momentum tensor, the supercurrent and the $\mathrm{SO}(8)$ current, respectively. They satisfy conservation laws and $(\gamma-)$ traceless conditions

$$
\begin{aligned}
& \partial_{\mu} T^{\mu \nu}=0, \quad \partial_{\mu} S^{\mu}=0, \quad \partial_{\mu} J^{\mu I J}=0, \\
& T_{\mu}{ }^{\mu}=0, \quad \gamma_{\mu} S^{\mu}=0 .
\end{aligned}
$$

From these quantities we can construct the $\operatorname{OSp}(8 \mid 4)$ charges

$$
Q_{T}[\xi]=\int d^{3} x \xi^{\nu} T_{\nu}^{0}, \quad Q_{S}[\epsilon]=\int d^{3} x \bar{\epsilon} S^{0}, \quad Q_{J}[\zeta]=\int d^{3} x \zeta^{I J} J^{0 I J},
$$

where $\xi^{\mu}, \epsilon$ and $\zeta^{I J}$ are solutions of (5.3). By (5.6) and (5.3) these charges are conserved. $R^{I}, M^{I J}, N^{I J K L}$ are quantities corresponding to the fields $\lambda^{I}, D^{I J}$, $E^{I J K L}$, respectively. $R^{I}, M^{I J}$ satisfy $\left(\Sigma\right.$-)traceless conditions $\Sigma^{I} R^{I}=0, M^{I I}=0$ and $N^{I J K L}$ is self-dual. 
Under the $\operatorname{OSp}(8 \mid 4)$ transformations in (5.2) these quantities transform into themselves. In particular, their superconformal transformations are

$$
\begin{aligned}
\delta_{S C} T_{\mu \nu}= & \frac{1}{2} \bar{\epsilon} \gamma_{\rho(\mu} \partial^{\rho} S_{\nu)}+2 \partial_{(\mu} \bar{\epsilon} S_{\nu)} \\
\delta_{S C} S_{\mu}= & \frac{1}{4} \gamma^{\nu} \epsilon T_{\mu \nu}-\frac{1}{16} \gamma^{\rho} \gamma_{\mu}{ }^{\sigma} \Sigma^{I J} \epsilon \partial_{\sigma} J_{\rho}^{I J}-\frac{1}{16} \Sigma^{I J}\left(\gamma_{\mu} \partial_{\nu}+3 \gamma_{\nu} \partial_{\mu}\right) \epsilon J^{\nu I J} \\
\delta_{S C} J_{\mu}^{I J}= & \frac{1}{4} \bar{\epsilon} \Sigma^{I J} S_{\mu}+\frac{1}{2 \sqrt{2}} \partial_{\nu}\left(\bar{\epsilon} \gamma_{\mu}{ }^{\nu} \Sigma^{[I} R^{J]}\right) \\
\delta_{S C} R^{I}= & \frac{1}{2 \sqrt{2}} \gamma^{\mu} \bar{\Sigma}^{J} \epsilon J_{\mu}^{I J}-\frac{1}{16 \sqrt{2}} \gamma^{\mu} \bar{\Sigma}^{I} \Sigma^{K L} \epsilon J_{\mu}^{K L}+\frac{1}{12 \sqrt{2}} \bar{\Sigma}^{J K L} \epsilon N^{I J K L} \\
& +\frac{1}{4 \sqrt{2}} \gamma^{\mu} \bar{\Sigma}^{J} \epsilon \partial_{\mu} M^{I J}+\frac{1}{6 \sqrt{2}} \gamma^{\mu} \bar{\Sigma}^{J} \partial_{\mu} \epsilon M^{I J}, \\
\delta_{S C} M^{I J}= & \frac{1}{\sqrt{2}} \bar{\epsilon} \Sigma^{(I} R^{J)}, \\
\delta_{S C} N^{I J K L}= & -\frac{1}{16 \sqrt{2}} \bar{\epsilon} \gamma^{\mu} \Sigma^{M} \bar{\Sigma}^{I J K L} \partial_{\mu} R^{M}-\frac{1}{48 \sqrt{2}} \partial_{\mu} \bar{\epsilon} \gamma^{\mu} \Sigma^{M} \bar{\Sigma}^{I J K L} R^{M} .
\end{aligned}
$$

We see that $T_{\mu \nu}, S_{\mu}$ and $J_{\mu}^{I J}$ transform into themselves except that $R^{I}$ appears in $\delta_{S C} J_{\mu}^{I J}$. However, this $R^{I}$ term does not contribute to transformations of the conserved charges (5.7). Indeed, we find that they transform as

$$
\begin{aligned}
\delta_{S C}\left(\epsilon_{1}\right) Q_{T}\left[\xi_{2}\right] & =Q_{S}\left[\epsilon_{3}\right], \\
\delta_{S C}\left(\epsilon_{1}\right) Q_{S}\left[\epsilon_{2}\right] & =Q_{T}\left[\xi_{3}\right]+Q_{J}\left[\zeta_{3}\right], \\
\delta_{S C}\left(\epsilon_{1}\right) Q_{J}\left[\zeta_{2}\right] & =Q_{S}\left[\epsilon_{3}^{\prime}\right],
\end{aligned}
$$

where the transformation parameters on the right-hand sides are

$$
\begin{aligned}
& \epsilon_{3}=\xi_{2}^{\mu} \partial_{\mu} \epsilon_{1}+\frac{1}{4} \partial_{\mu} \xi_{2 \nu} \gamma^{\mu \nu} \epsilon_{1}-\frac{1}{6} \partial_{\mu} \xi_{2}^{\mu} \epsilon_{1}, \quad \epsilon_{3}^{\prime}=-\frac{1}{4} \zeta_{2}^{I J} \Sigma^{I J} \epsilon_{1} \\
& \xi_{3}^{\mu}=\frac{1}{4} \bar{\epsilon}_{2} \gamma^{\mu} \epsilon_{1}, \quad \zeta_{3}^{I J}=-\frac{1}{24}\left(\bar{\epsilon}_{2} \Sigma^{I J} \gamma^{\mu} \partial_{\mu} \epsilon_{1}-\bar{\epsilon}_{1} \Sigma^{I J} \gamma^{\mu} \partial_{\mu} \epsilon_{2}\right)
\end{aligned}
$$

It can be shown that these parameters satisfy (5.3), i.e., $\epsilon_{3}, \epsilon_{3}^{\prime}$ are conformal Killing spinors, $\xi_{3}^{\mu}$ is a conformal Killing vector and $\zeta_{3}^{I J}$ is a constant. The conserved charges (5.7) are transformed into themselves also by the conformal and $\mathrm{SO}(8)$ transformations. 


\section{Discussion}

In this paper we obtained local transformation laws of the $D=3, \mathcal{N}=8$ conformal supergravity. There are two cases depending on the parameter $\eta= \pm 1$ appearing in the (anti) self-duality conditions on the scalar fields. In both cases the commutator algebra of the local transformations closes off-shell on the conformal supergravity fields. In the $\eta=+1$ case we coupled the conformal supergravity to the BLG theory and obtained local transformation laws and an invariant Lagrangian of the coupled theory. The commutator algebra closes also on the BLG fields when their field equations are used.

On the other hand, in the $\eta=-1$ case we did not succeed in coupling the conformal supergravity to the BLG theory. We found a difficulty in a commutator of local supertransformations. Assuming that the local supertransformation laws of the BLG fields are given by

$$
\delta_{Q} \Psi_{i}=\frac{1}{2 \sqrt{2}} \gamma^{\mu} \bar{\Sigma}^{I} \epsilon \hat{D}_{\mu} X_{i}^{I}+\frac{1}{12 \sqrt{2}} f_{i}^{j k l} X_{j}^{J} X_{k}^{K} X_{l}^{L} \bar{\Sigma}^{J K L} \epsilon+\frac{1}{2} \bar{\Sigma}^{I} \epsilon E^{I J} X_{i}^{J}
$$

and $\delta_{Q} X_{i}^{I}, \delta_{Q} \tilde{A}_{\mu}{ }^{j}{ }_{i}$ in (4.3) , we found an extra term in the commutation relation

$$
\left[\delta_{Q}\left(\epsilon_{1}\right), \delta_{Q}\left(\epsilon_{2}\right)\right] X_{i}^{I}=\cdots-\frac{1}{2 \sqrt{2}} \bar{\epsilon}_{2} \Sigma^{K(I} \epsilon_{1} E^{J) K} X_{i}^{J}
$$

where $\cdots$ denote the expected local transformation terms in (4.10). Therefore, this commutator does not close. A change of the coefficient in the last term of (6.1) or an addition of terms of the form $\bar{\Sigma}^{I} \epsilon X^{I} X^{J} X^{J}$ to (6.1) does not improve the situation. Another puzzling point is on the supercurrent multiplet of the BLG theory in a flat background. If one could find a coupling of the $\eta=-1$ conformal supergravity, one would obtain a supercurrent multiplet different from (5.5). In particular, $M^{I J}$, $N^{I J K L}$ would be replaced by something like $M^{I J K L}, N^{I J}$. Since these multiplets are those of the original BLG theory without coupling to the conformal supergravity, we would obtain two different supercurrent multiplets for the same one theory. These observations seem to suggest that it is not possible to couple the $\eta=-1$ off-shell conformal supergravity to the BLG theory.

The $D=3, \mathcal{N}=8$ conformal supergravity and its coupling to the BLG theory were previously discussed in [16 19] in both of the component field formulation and the superspace formulation. Let us compare these works with ours. In the component field formulation [16, 17] the on-shell conformal supergravity multiplet, 
which consists of a dreibein $e_{\mu}{ }^{a}$, Rarita-Schwinger fields $\psi_{\mu}^{\alpha}$ and $\mathrm{SO}(8)$ gauge fields $B_{\mu}^{I J}$, was used. The Lagrangian and the local supertransformations of these fields were obtained. The conformal supergravity multiplet has a kinetic term in the Lagrangian and is dynamical. The commutator algebra closes only on-shell, i.e., when field equations of the conformal supergravity fields are used. In the superspace formulation [18, 19, 17] the conformal supergravity multiplet is represented by superfields. The superfield of central importance is the "super Cotton tensor", which contains the Cotton tensor for the gravitational field, its superpartner (Cottino) for the Rarita-Schwinger fields and the field strength of the $\mathrm{SO}(8)$ gauge fields as component fields. In addition, the super Cotton tensor also contains spinor and scalar fields $\lambda^{\alpha \beta \gamma}, D^{\alpha \beta \gamma \delta}, E^{\alpha \beta \gamma \delta}$. Its leading component field is the scalar field $E^{\alpha \beta \gamma \delta}$, which is either self-dual or anti self-dual. Therefore, this formulation corresponds to the off-shell conformal supergravity multiplet as in our approach. However, when it is coupled to the BLG matter theory, the field equations of the matter fields were given only when the conformal supergravity fields are on-shell, i.e., they satisfy their field equations [17]. In particular, the spinor and scalar fields are not independent fields but are expressed in terms of the matter fields as determined by (4.25), (4.26) of [17]. Then, the superspace formulation reduces to the on-shell component field formulation.

To compare these results with ours we first suppose that our results on the offshell conformal supergravity multiplet in section 3 correspond to a component field form of the superspace formulation in [18, 19, 17] although we have not shown it explicitly. As for the coupling to the BLG theory we note that in the superspace formulation [17] the coupling was studied only when $E^{\alpha \beta \gamma \delta}$ is self-dual and not anti self-dual. This corresponds to our $\eta=-1$ case as can be seen by comparing (D.13), (D.14) of [17] and our (3.17) with $D$ and $E$ interchanged for $\eta=-1$. Although we could not find a coupled theory for $\eta=-1$, the supertransformations of the conformal supergravity multiplet were obtained and are given in (3.24). Comparing the supertransformations of the fields $e_{\mu}{ }^{a}, \psi_{\mu}, B_{\mu}^{I J}$ in (3.24) and those in (2.20)- 
(2.22) of [17] we see that they are the same if we assume*

$$
\begin{aligned}
\lambda^{I} & =\frac{1}{8} g\left(\Psi^{i} X_{i}^{I}-\frac{1}{8} \bar{\Sigma}^{I} \Sigma^{J} \Psi^{i} X_{i}^{J}\right), \\
E^{I J} & =\frac{1}{4 \sqrt{2}} g\left(X_{i}^{I} X^{i J}-\frac{1}{8} \delta^{I J} X_{i}^{K} X^{i K}\right),
\end{aligned}
$$

where $g$ is a conformal gravitational coupling constant used in [17]. Furthermore, (6.3) appear as field equations for the conformal supergravity multiplet in the superspace formulation $((4.25),(4.26)$ of [17]). Therefore, our results for $\eta=-1$ are consistent with those of [16,17] as far as the transformations of the conformal supergravity multiplet are concerned. Although we could not find a coupled theory for the off-shell conformal supergravity multiplet, it is possible to construct a coupled theory for the on-shell multiplet as was done in [16, 17].

A coupling of the BLG theory to the $\eta=+1$ conformal supergravity multiplet was not considered in the superspace formulation in [17]. It would be interesting to study a coupling to the $\eta=+1$ multiplet in the superspace formulation and compare it with our results in section 4 . In connection with this it would be also interesting to see whether one can construct a theory in which the $\eta=+1$ conformal supergravity multiplet is dynamical and can eliminate the auxiliary spinor and scalar fields in terms of the matter fields by using field equations to obtain an on-shell multiplet. If such a theory is possible, the second equation of (6.3) will be replaced by an equation for $E^{I J K L}$. One may guess that it is something like $E^{I J K L} \sim g N^{I J K L}\left(X^{2}\right)^{-1}$, where $N^{I J K L}$ is given in (5.5) and the factor $\left(X^{2}\right)^{-1}$ is needed to match the Weyl weight. Then, on-shell supertransformations will have different forms from those of [16, 17].

Finally, $D=3$ conformal supergravity multiplets with lower $\mathcal{N}$ supersymmetries can be obtained from the $\mathcal{N}=8$ multiplet given in section 3 by consistent truncations, i.e., by setting some of the fields to zero in such a way that a part of supersymmetries is preserved. Their local transformation laws are easily obtained from those of the $\mathcal{N}=8$ multiplet. For instance, a truncation to $\mathcal{N}=6$ can be obtained as follows. In section 3 we used $\mathrm{SO}(8)$ negative chirality indices $\alpha, \beta, \gamma, \cdots=1,2, \cdots, 8$ for the $\mathcal{N}=8$ conformal supergravity multiplet as given in Table 1 and the parameters of the super and super Weyl transformations $\epsilon^{\alpha}, \eta^{\alpha}$. Alternatively, we can consistently replace all these indices by $\mathrm{SO}(8)$ vector indices

*The right-hand sides of these equations are proportional to $R^{I}$ and $M^{I J}$ in the supercurrent multiplet of the $\eta=+1$ theory (5.5), respectively. It seems natural then to also assume that $D^{I J K L}$ is proportional to $N^{I J K L}$ in (5.5). 
$I, J, K, \cdots=1,2, \cdots, 8$. Then, the conformal supergravity multiplet and the transformation parameters become $e_{\mu}{ }^{a}, \psi_{\mu}^{I}, B_{\mu}^{I J}, \lambda^{I J K}, D^{I J K L}, E^{I J K L}$ and $\epsilon^{I}, \eta^{I}$. The truncation to $\mathcal{N}=6$ is given by setting $\psi_{\mu}^{I^{\prime}}=0, B_{\mu}^{I J^{\prime}}=0, \lambda^{I J K^{\prime}}=0, D^{I J K L^{\prime}}=0$, $E^{I J K L^{\prime}}=0$, where $I, J, K, \cdots=1,2, \cdots, 6$ and $I^{\prime}, J^{\prime}, K^{\prime}, \cdots=7,8$. These conditions are invariant under the super and super Weyl transformations (3.3), (3.4) when $\epsilon^{I^{\prime}}=0, \eta^{I^{\prime}}=0$. The remaining independent fields are

$$
e_{\mu}{ }^{a}, \quad \psi_{\mu}^{I}, \quad B_{\mu}^{I J}, \quad B_{\mu}^{78}, \quad \lambda^{I J K}, \quad \lambda^{I 78}, \quad D^{I J K L}, \quad E^{I J K L},
$$

where $I, J, K, \cdots=1,2, \cdots, 6$. The fields $D^{I J 78}, E^{I J 78}$ are related to $D^{I J K L}, E^{I J K L}$ in (6.4) by the self-duality conditions (3.1). The $\mathrm{SO}(8)$ gauge transformation is reduced to $\mathrm{SO}(6) \times \mathrm{U}(1)$, whose gauge fields are $B_{\mu}^{I J}$ and $B_{\mu}^{78}$ in (6.4). The fields in (6.4) constitute the $\mathcal{N}=6$ conformal supergravity multiplet as given by the superspace formulation [18, 17]. The $\mathcal{N}=6$ multiplet is interesting since it can be coupled to the ABJM theory [27] of multiple M2-branes. A coupling of the dynamical $\mathcal{N}=6$ conformal supergravity to the ABJM theory was already studied in the on-shell component field formulation [28,29] and in the off-shell superspace formulation [17]. For $\mathcal{N} \leq 4$, off-shell conformal supergravities and their couplings to matter multiplets were studied in the superspace formulation in [30]. We can use the lower $\mathcal{N}$ off-shell conformal supergravity multiplets obtained by the truncations from $\mathcal{N}=8$ to study their coupling to matter multiplets in terms of component fields.

\section{Acknowledgments}

M.N. would like to thank Theoretical High Energy Physics Group of Bielefeld University, where part of this work was done, for hospitality.

\section{A. Notations and Conventions}

Three-dimensional world and local Lorentz indices are denoted by $\mu, \nu, \cdots=0,1,2$ and $a, b, \cdots=0,1,2$, respectively. A flat metric is $\eta_{a b}=\operatorname{diag}(-1,+1,+1)$ and the antisymmetric symbol $\epsilon^{a b c}$ is chosen as $\epsilon^{012}=+1$. Symmetrization and antisymmetrization of indices with weight one are denoted as $(a b \cdots)$ and $[a b \cdots]$, respectively. Three-dimensional gamma matrices are denoted by $\gamma^{a}$. Their antisymmetrized products $\gamma^{a b}=\gamma^{[a} \gamma^{b]}, \gamma^{a b c}=\gamma^{[a} \gamma^{b} \gamma^{c]}$ satisfy

$$
\gamma^{a b c}=-\epsilon^{a b c}, \quad \gamma^{a b}=-\epsilon^{a b c} \gamma_{c}, \quad \gamma^{a}=\frac{1}{2} \epsilon^{a b c} \gamma_{b c} .
$$


There are three eight-dimensional representations of $\mathrm{SO}(8)$ : a vector representation $\boldsymbol{8}_{v}$, a negative chirality Weyl spinor representation $\boldsymbol{8}_{s}$ and a positive chirality Weyl spinor representation $\boldsymbol{8}_{c}$. Indices of these representations are denoted by $I, J, \cdots=1,2, \cdots, 8 ; \alpha, \beta, \cdots=1,2, \cdots, 8$ and $\dot{\alpha}, \dot{\beta}, \cdots=1,2, \cdots, 8$, respectively. For these indices upper and lower indices are not distinguished.

\section{B. $\mathrm{SO}(8)$ identities}

In this Appendix we summarize some properties of the matrices $\Sigma^{I}$ and $\bar{\Sigma}^{I}=\left(\Sigma^{I}\right)^{T}$ introduced in (3.11). From the anti-commutation relation of the $\mathrm{SO}(8)$ gamma matrices $\left\{\Gamma^{I}, \Gamma^{J}\right\}=2 \delta^{I J}$ they satisfy

$$
\begin{aligned}
& \left(\Sigma^{I}\right)_{\alpha \dot{\gamma}}\left(\Sigma^{J}\right)_{\beta \dot{\gamma}}+\left(\Sigma^{J}\right)_{\alpha \dot{\gamma}}\left(\Sigma^{I}\right)_{\beta \dot{\gamma}}=2 \delta^{I J} \delta_{\alpha \beta}, \\
& \left(\Sigma^{I}\right)_{\gamma \dot{\alpha}}\left(\Sigma^{J}\right)_{\gamma \dot{\beta}}+\left(\Sigma^{J}\right)_{\gamma \dot{\alpha}}\left(\Sigma^{I}\right)_{\gamma \dot{\beta}}=2 \delta^{I J} \delta_{\dot{\alpha} \dot{\beta}} .
\end{aligned}
$$

They also satisfy

$$
\left(\Sigma^{I}\right)_{\alpha \dot{\alpha}}\left(\Sigma^{I}\right)_{\beta \dot{\beta}}+\left(\Sigma^{I}\right)_{\alpha \dot{\beta}}\left(\Sigma^{I}\right)_{\beta \dot{\alpha}}=2 \delta_{\alpha \beta} \delta_{\dot{\alpha} \dot{\beta}}
$$

as can be shown by using the Fierz identity. Similarity of these three equations is a consequence of the triality of three eight-dimensional representations of $\mathrm{SO}(8)$. We choose sign conventions of these matrices such that

$$
\begin{aligned}
& \left(\Sigma^{\left[I_{1}\right.}\right)_{\alpha_{1} \dot{\alpha}_{2}}\left(\bar{\Sigma}^{I_{2}}\right)_{\dot{\alpha}_{2} \alpha_{3}}\left(\Sigma^{I_{3}}\right)_{\alpha_{3} \dot{\alpha}_{4}} \cdots\left(\bar{\Sigma}^{\left.I_{8}\right]}\right)_{\dot{\alpha}_{8} \alpha_{9}}=-\delta_{\alpha_{1} \alpha_{9}} \epsilon^{I_{1} \cdots I_{8}} \\
& \left(\Sigma^{I_{1}}\right)_{\left[\alpha_{1}\right.} \dot{\alpha}_{2}\left(\Sigma^{I_{2}}\right)_{\alpha_{2}}{ }^{\dot{\alpha}_{2}}\left(\Sigma^{I_{2}}\right)_{\alpha_{3}} \dot{\alpha}_{3} \cdots\left(\Sigma^{I_{5}}\right)_{\left.\alpha_{8}\right]} \dot{\alpha}_{5}=\delta^{I_{1} I_{5}} \epsilon_{\alpha_{1} \cdots \alpha_{8}}
\end{aligned}
$$

are satisfied.

We denote antisymmetrized products of these matrices as

$$
\begin{aligned}
& \Sigma^{I J}=\Sigma^{[I} \bar{\Sigma}^{J]}, \quad \Sigma^{I J K}=\Sigma^{[I} \bar{\Sigma}^{J} \Sigma^{K]}, \quad \cdots, \\
& \bar{\Sigma}^{I J}=\bar{\Sigma}^{[I} \Sigma^{J]}, \quad \bar{\Sigma}^{I J K}=\bar{\Sigma}^{[I} \Sigma^{J} \bar{\Sigma}^{K]}, \quad \cdots \text {. }
\end{aligned}
$$


In general the leftmost matrix in $\Sigma^{I J \cdots}$ is $\Sigma^{I}$ while that in $\bar{\Sigma}^{I J \cdots}$ is $\bar{\Sigma}^{I}$. By the first equation of (B.3) these matrices satisfy

$$
\begin{aligned}
& \Sigma^{I_{1} \cdots I_{4}}=-\frac{1}{4 !} \epsilon^{I_{1} \cdots I_{8}} \Sigma^{I_{5} \cdots I_{8}}, \\
& \Sigma^{I_{1} \cdots I_{5}}=\frac{1}{3 !} \epsilon^{I_{1} \cdots I_{8}} \Sigma^{I_{6} I_{7} I_{8}}, \\
& \Sigma^{I_{1} \cdots I_{6}}=\frac{1}{2} \epsilon^{I_{1} \cdots I_{8}} \Sigma^{I_{7} I_{8}}, \\
& \Sigma^{I_{1} \cdots I_{7}}=-\epsilon^{I_{1} \cdots I_{8}} \Sigma^{I_{8}}, \\
& \Sigma^{I_{1} \cdots I_{8}}=-\epsilon^{I_{1} \cdots I_{8}}
\end{aligned}
$$

and similar identities for $\bar{\Sigma}^{I J \cdots}$ with opposite signs on the right-hand sides. In particular, $\Sigma^{I_{1} \cdots I_{4}}$ and $\bar{\Sigma}^{I_{1} \cdots I_{4}}$ are anti self-dual and self-dual, respectively. Other useful identities are

$$
\begin{aligned}
\Sigma^{M} \bar{\Sigma}^{M} & =8, & \Sigma^{M N} \Sigma^{M N} & =-56, \\
\Sigma^{M} \bar{\Sigma}^{I} \Sigma^{M} & =-6 \Sigma^{I}, & \Sigma^{M N} \Sigma^{I} \bar{\Sigma}^{M N} & =-28 \Sigma^{I}, \\
\Sigma^{M} \bar{\Sigma}^{I J} \bar{\Sigma}^{M} & =4 \Sigma^{I J}, & \Sigma^{M N} \Sigma^{I J} \Sigma^{M N} & =-8 \Sigma^{I J}, \\
\Sigma^{M} \bar{\Sigma}^{I J K} \Sigma^{M} & =-2 \Sigma^{I J K}, & \Sigma^{M N} \Sigma^{I J K} \bar{\Sigma}^{M N} & =4 \Sigma^{I J K}, \\
\Sigma^{M} \bar{\Sigma}^{I J K L} \bar{\Sigma}^{M} & =0, & \Sigma^{M N} \Sigma^{I J K L} \Sigma^{M N} & =8 \Sigma^{I J K L} .
\end{aligned}
$$

Bilinears of two $\mathrm{SO}(1,2)$ Majorana spinors $\psi_{1}^{\alpha}$ and $\psi_{2}^{\alpha}$ in the representation $\boldsymbol{8}_{s}$ of $\mathrm{SO}(8)$ have symmetries

$$
\begin{aligned}
\bar{\psi}_{1} \psi_{2} & =\bar{\psi}_{2} \psi_{1}, & \bar{\psi}_{1} \gamma^{a} \psi_{2} & =-\bar{\psi}_{2} \gamma^{a} \psi_{1}, \\
\bar{\psi}_{1} \Sigma^{I J} \psi_{2} & =-\bar{\psi}_{2} \Sigma^{I J} \psi_{1}, & \bar{\psi}_{1} \gamma^{a} \Sigma^{I J} \psi_{2} & =\bar{\psi}_{2} \gamma^{a} \Sigma^{I J} \psi_{1}, \\
\bar{\psi}_{1} \Sigma^{I J K L} \psi_{2} & =\bar{\psi}_{2} \Sigma^{I J K L} \psi_{1}, & \bar{\psi}_{1} \gamma^{a} \Sigma^{I J K L} \psi_{2} & =-\bar{\psi}_{2} \gamma^{a} \Sigma^{I J K L} \psi_{1} .
\end{aligned}
$$

The Fierz identity for spinors $\psi_{1}^{\alpha}, \cdots, \psi_{4}^{\alpha}$ can be written as

$$
\begin{aligned}
\bar{\psi}_{1} \psi_{2} \bar{\psi}_{3} \psi_{4}= & -\frac{1}{16}\left(\bar{\psi}_{1} \psi_{4} \bar{\psi}_{3} \psi_{2}+\bar{\psi}_{1} \gamma^{a} \psi_{4} \bar{\psi}_{3} \gamma_{a} \psi_{2}\right) \\
& +\frac{1}{32}\left(\bar{\psi}_{1} \Sigma^{I J} \psi_{4} \bar{\psi}_{3} \Sigma^{I J} \psi_{2}+\bar{\psi}_{1} \gamma^{a} \Sigma^{I J} \psi_{4} \bar{\psi}_{3} \gamma_{a} \Sigma^{I J} \psi_{2}\right) \\
& -\frac{1}{32 \cdot 4 !}\left(\bar{\psi}_{1} \Sigma^{I J K L} \psi_{4} \bar{\psi}_{3} \Sigma^{I J K L} \psi_{2}+\bar{\psi}_{1} \gamma^{a} \Sigma^{I J K L} \psi_{4} \bar{\psi}_{3} \gamma_{a} \Sigma^{I J K L} \psi_{2}\right) .
\end{aligned}
$$


When totally antisymmetric tensors $S^{I J K L}, S^{I J K L}$ are self-dual and $A^{I J K L}$, $A^{\prime I J K L}$ are anti self-dual, the following identities are satisfied:

$$
\begin{aligned}
S^{I J K L} A^{I J K L} & =0 \\
S^{I K L M} A^{J K L M} & =S^{J K L M} A^{I K L M}, \\
S^{I J M N} A^{K L M N}+S^{K L M N} A^{I J M N} & =-\frac{4}{3} S^{M N P[I} \delta^{J][L} A^{K] M N P}, \\
S^{K L M(I} S^{\prime J) K L M} & =-\frac{1}{8} \delta^{I J} S^{K L M N} S^{\prime K L M N} \\
A^{K L M(I} A^{\prime J) K L M} & =-\frac{1}{8} \delta^{I J} A^{K L M N} A^{\prime K L M N} .
\end{aligned}
$$

\section{References}

[1] J. Bagger and N. Lambert, "Modeling multiple M2-branes," Phys. Rev. D75 (2007) 045020 arXiv:hep-th/0611108.

[2] A. Gustavsson, "Algebraic structures on parallel M2 branes," Nucl. Phys. B811 (2009) 66 [arXiv:0709.1260 [hep-th]].

[3] J. Bagger and N. Lambert, "Gauge symmetry and supersymmetry of multiple M2-branes," Phys. Rev. D77 (2008) 065008 [arXiv:0711.0955 [hep-th]].

[4] J. Bagger, N. Lambert, S. Mukhi and C. Papageorgakis, "Membranes in Mtheory," arXiv:1203.3546 [hep-th].

[5] J. Maldacena, "The large $N$ limit of superconformal field theories and supergravity," Adv. Theor. Math. Phys. 2 (1998) 231 arXiv:hep-th/9711200.

[6] S.S. Gubser, I.R. Klebanov and A.M. Polyakov, "Gauge theory correlators from non-critical string theory," Phys. Lett. B428 (1998) 105 arXiv:hep-th/9802109.

[7] E. Witten, "Anti de Sitter space and holography," Adv. Theor. Math. Phys. 2 (1998) 253 arXiv:hep-th/9802150].

[8] S. Ferrara, C. Fronsdal and A. Zaffaroni, "On $\mathcal{N}=8$ supergravity on $\mathrm{AdS}_{5}$ and $\mathcal{N}=4$ superconformal Yang-Mills theory," Nucl. Phys. B532 (1998) 153 arXiv:hep-th/9802203. 
[9] H. Liu and A.A. Tseytlin, " $D=4$ super Yang-Mills, $D=5$ gauged supergravity, and $D=4$ conformal supergravity," Nucl. Phys. B533 (1998) 88 arXiv:hep-th/9804083.

[10] M. Nishimura and Y. Tanii, "Supersymmetry in the AdS/CFT correspondence," Phys. Lett. B446 (1999) 37 [arXiv:hep-th/9810148].

[11] M. Nishimura and Y. Tanii, "Super Weyl anomalies in the AdS/CFT correspondence," Int. J. Mod. Phys. A14 (1999) 3731 arXiv:hep-th/9904010].

[12] M. Nishimura and Y. Tanii, "Local symmetries in the $\mathrm{AdS}_{7} / \mathrm{CFT}_{6}$ correspondence," Mod. Phys. Lett. A14 (1999) 2709 arXiv:hep-th/9910192.

[13] M. Nishimura, "Conformal supergravity from the AdS/CFT correspondence," Nucl. Phys. B588 (2000) 471 arXiv:hep-th/0004179].

[14] E. Bergshoeff, M. de Roo and B. de Wit, "Extended conformal supergravity," Nucl. Phys. B182 (1981) 173.

[15] G. Festuccia and N. Seiberg, "Rigid supersymmetric theories in curved superspace," JHEP 1106 (2011) 114 [arXiv:1105.0689 [hep-th]].

[16] U. Gran and B.E.W. Nilsson, "Three-dimensional $\mathcal{N}=8$ conformal supergravity and its coupling to BLG M2-branes," JHEP 0903 (2009) 074 arXiv:0809.4478 [hep-th]].

[17] U. Gran, J. Greitz, P. Howe and B.E.W. Nilsson, "Topologically gauged superconformal Chern-Simons matter theories," JHEP 1212 (2012) 046 arXiv:1204.2521 [hep-th]].

[18] P.S. Howe, J.M. Izquierdo, G. Papadopoulos and P.K. Townsend, "New supergravities with central charges and Killing spinors in $(2+1)$-dimensions," Nucl. Phys. B467 (1996) 183 arXiv:hep-th/9505032.

[19] M. Cederwall, U. Gran and B.E.W. Nilsson, " $D=3, \mathcal{N}=8$ conformal supergravity and the Dragon window," JHEP 1109 (2011) 101 arXiv:1103.4530 [hep-th]].

[20] E.A. Bergshoeff, O. Hohm, J. Rosseel and P.K. Townsend, "On maximal massive 3D supergravity," Class. Quant. Grav. 27 (2010) 235012 [arXiv:1007.4075 [hep-th]]. 
[21] B. de Wit and H. Nicolai, "N = 8 supergravity," Nucl. Phys. B208 (1982) 323.

[22] E. Cremmer and B. Julia, "The SO(8) supergravity," Nucl. Phys. B159 (1979) 141.

[23] R. Slansky, "Group theory for unified model building," Phys. Rept. 79 (1981) 1.

[24] M.B. Green, J.H. Schwarz and L. Brink, "Superfield theory of type II superstrings," Nucl. Phys. B219 (1983) 437.

[25] S. Ferrara and B. Zumino, "Transformation properties of the supercurrent," Nucl. Phys. B87 (1975) 207.

[26] M.A. Bandres, A.E. Lipstein and J.H. Schwarz, "N $\mathcal{N}=8$ Superconformal ChernSimons theories," JHEP 0805 (2008) 025 [arXiv:0803.3242 [hep-th]].

[27] O. Aharony, O. Bergman, D.L. Jafferis and J. Maldacena, "N $\mathcal{N}=6$ superconformal Chern-Simons-matter theories, M2-branes and their gravity duals," JHEP 0810 (2008) 091 [arXiv:0806.1218 [hep-th]].

[28] X. Chu and B. E. W. Nilsson, "Three-dimensional topologically gauged $\mathcal{N}=6$ ABJM type theories," JHEP 1006 (2010) 057 [arXiv:0906.1655 [hep-th]].

[29] X. Chu, H. Nastase, B.E.W. Nilsson and C. Papageorgakis, "Higgsing M2 to D2 with gravity: $N=6$ chiral supergravity from topologically gauged ABJM theory," JHEP 1104 (2011) 040 [arXiv:1012.5969 [hep-th]].

[30] S.M. Kuzenko, U. Lindström and G. Tartaglino-Mazzucchelli, "Off-shell supergravity-matter couplings in three dimensions," JHEP 1103 (2011) 120 arXiv:1101.4013 [hep-th]]. 